\title{
A dual-energy-demand-indices-based evaluation procedure of damage-control frame structures with energy dissipation fuses
}

\author{
Ke Ke ${ }^{\mathrm{a}, \mathrm{b}}$, Michael C.H. Yam ${ }^{\mathrm{b}}$, Shuizhou Ke ${ }^{\mathrm{c}, *}$ \\ ${ }^{a}$ State Key Laboratory of Disaster Reduction in Civil Engineering, Tongji University, Shanghai \\ 200092, China \\ ${ }^{\mathrm{b}}$ Department of Building and Real Estate, The Hong Kong Polytechnic University, Hung Hom, \\ Kowloon, Hong Kong, China \\ ${ }^{c}$ College of Civil Engineering, Hunan University, Changsha 410082, China
}

\begin{abstract}
The primary objective of this research is to extend energy balance concept in seismic behavior evaluation of low-to-medium rise frame systems with energy dissipation fuses dominated by racking deformations. At its core, energy demand indices under ground motions considering both peak responses and cumulative responses are quantified to develop a dual-energy-demand-indices-based procedure for damage-control behavior evaluation. Firstly, based on the experimentally validated hysteretic feature and representative ground motion ensembles, a parametric study is conducted considering the demand indices of the energy factor and the cumulative ductility. Results of the indices distribution and dispersion are presented in detail. Subsequently, a stepwise procedure that accounts for the peak demand and the cumulative demand is constructed. Then, the procedure is applied in prototype structures for validation. Results indicate that the consideration of dual energy demand indices is necessary for evaluation of systems with fuses, and these indices are influenced by structural nonlinear parameters and ground motions properties. The procedure based on the dual energy demand indices can be used to evaluate the structural damage-control behavior with satisfactory accuracy considering the peak response, the cumulative response, and the energy distribution along stories.
\end{abstract}

Keywords: energy demand indices; energy factor; cumulative ductility; pushover analysis; damage-control; structural fuse.

* Corresponding author. Tel.: +86-731-88823273;

Email address: ksz@hnu.edu.cn (S. Ke) 


\section{Introduction}

Although nonlinear response history analysis (NL-RHA) is the most rigorous procedure to analyze structural responses of structures subjected to ground motions, static design and evaluation procedures are preferred as the first step in engineering practice for their conceptual simplicity and computational efficiency. Fundamentally, a successful seismic design by static procedures requires the appropriate determination of demand indices of a structure subjected to ground motions. In this respect, many efforts have been made for quantifying inelastic seismic demand indices in recent decades. In particular, indicators representing seismic demands in terms of strength [1-4] and displacement [5-8] have been extensively investigated as the foundation of the widely used force-based and displacement-based procedures. Since the satisfactory effectiveness of these indices for estimating structural seismic demands was validated, they have been documented in design provisions [9-10] and evaluation methods [11-12]. More recently, procedures including multiple demand indices were developed to account for multiple performance objectives. For instance, Guo and Christopoulos [13-14] proposed the performance spectra employing the demand indices of force and displacement simultaneously, and applied them in supplemental damping system with energy dissipation dampers. In parallel with these investigations, research works suggest that the inelastic seismic design and evaluation can also be conducted with the application of energy balance concept. An energy factor [15-16] derived from the modified Housner equation $[15,17]$ can serve as a reliable demand index to quantify the inelastic demand of systems subjected to ground motions, and the feature of strength and displacement can be favorably quantified by this single indicator, greatly 
facilitating a design or evaluation procedure. Therefore, interest has been directed to the seismic energy factor and energy-factor-based seismic design or evaluation procedures. For instance, Zhai et al. [18] developed formulations of the energy factor of single-degree-of-freedom systems under near-fault pulse-like ground motions for practical applications. Sahoo and Chao [19] derived the design base shear for buckling-restrained braced frames and developed a design procedure using the energy factor associated with the Newmark and Hall spectra [1]. Likewise, Peckcan et al. [20-21] developed design frameworks for innovative truss girder frame system with the energy factor. Heidari and Gharehbaghi [22] applied the energy factor as the demand index to develop a refined procedure considering truss girder frame systems with energy dissipation devices. Kharmale and Ghosh [23] constructed a design procedure of the steel plate shear wall structures with the energy factor denoting the demand. Jiang et al. [24] adopted the energy factor to determine the seismic energy demand of systems and developed an energy-based multi-mode pushover analysis procedure. In general, these investigations have demonstrated the tremendous potential of the energy factor in seismic engineering. However, it should be noted that the energy factor is a quantity considering the peak response of the system since the energy balance equation is established based on the absorbed energy determined from the skeleton pushover curve. In this regard, applying this single indicator as the demand index might lead to unconservative estimates since failure might occur due to cumulative damage [25-32] even when the peak response is not significant, particularly for the recently expanded innovative systems installed with energy dissipation fuses [13-14, 33-39], since the fuse elements are designated as the primary 
source of plastic energy dissipation. On the other hand, although current methods established based on cumulative absorbed energy of a system essentially cover the peak response, the dependency of demand indices on ground motions would be amplified as the cumulative effect may vary dramatically for different ground motions, which might conceal some basic rules if only the quantities representing the cumulative demand is used in design and evaluation. For instance, the classical equal-energy and equal-displacement rules proposed by Newmark and Hall $[1,15]$, which are of great values for seismic design, might not be revealed directly if only the cumulative energy is considered, since these rules were obtained based on skeleton pushover curves. Moreover, for a system equipped with energy dissipation fuses, the nonlinear behavior of the entire system is altered, leading to the remarkable post-yielding stiffness ratio after the yielding of fuses is triggered, but the available formulas of the widely used energy demand indices (e.g. energy factor, cumulative ductility) were generally established on a perfectly elasto-plastic (EP) model or a bilinear model of negligible post-yielding stiffness ratio. Thus, improvement should be made when applying the energy balance concept in seismic demand quantification of the innovative damage-control systems with energy dissipation fuses.

In this research, to evaluate the damage-control behavior of low-to-medium rise frame structures with energy dissipation fuses dominated by racking deformations (global shear deformations), two energy demand indices, the energy factor and the cumulative ductility, are adopted to construct an energy-balance-concept-based damage-control evaluation procedure, considering the peak demand and the cumulative seismic demand, 
simultaneously. Firstly, the hysteretic feature of the systems is quantified and validated based on a recent experimental investigation. For low-to-medium rise frame structures which are dominated by racking deformations under earthquakes without being appreciably influenced by higher vibration modes, recent research works $[8,12,15]$ indicate that the seismic response of the entire structure as a multi-degree-of-freedom (MDOF) system can be reasonably quantified by an equivalent single-degree-of-freedom (SDOF) system considering the fundamental vibration mode. Thus, the energy factor and the cumulative ductility are analyzed with SDOF systems with the validated hysteretic feature below the defined resilience threshold, considering various combinations of nonlinear parameters under ground motion ensembles to establish a parametric study. Subsequently, an energy-balance-concept-based approach defined as dual-energy-demand-indices-based (DEB) procedure is constructed for evaluating the seismic demands of frame structures with energy dissipation fuses. Lastly, case studies are conducted to validate the attractiveness of the procedure. It is noted that although the DEB procedure does not involve the quantification of damage levels of fuse elements, it can be utilized to reasonably estimate the seismic demand of damage-control systems with energy dissipation fuses, and the proposed framework can be used as the sound basis for a complete energy-based seismic design method associated with research works quantifying the energy dissipation capacity of structural elements [28].

\section{Damage-control behavior and energy demand indices of systems with fuses}

\subsection{Hysteretic behavior and experimental validation}

For a damage-control system with energy dissipation fuses, the fuse elements are 
expected to yield before inception of yielding of the remaining structure defined as the primary system [35]. Therefore, the structural hysteretic behavior is transformed in different states, as schematically illustrated in Fig. 1. The requirements considering the damage-control behavior are given as follows: (1) The primary system should stay elastic under expected ground motions with damages restricted in the energy dissipation fuses; (2) The energy dissipation fuses should provide a stable source of plastic energy dissipation to satisfy the cumulative demand without occurrence of failure. A damage-control core (DCC) following the classical bilinear kinematic hysteretic law can be extracted from the hysteretic curve. Because of the elastic behavior of the primary system, the post-yielding stiffness ratio of the DCC is generally significant [34], and the deformation corresponding to inception of yielding of the primary system is defined as the resilience threshold.

The damage-control behavior and hysteretic feature have been validated by a recent large-scale experimental study of a steel moment resisting frame (MRF) equipped with energy dissipation fuse beams. Specifically, preselected bays in a frame structure were designated as energy dissipation bays with fuse beams, and the remaining frames were expected to stay damage-free in a wider deformation range. In a steel MRF, the novel concept can be readily realized by applying members with different yield strengths. For the purpose of clarification, brief information on the experimental study is illustrated in Fig. 2. As a representative damage-control frame system with energy dissipation fuses, the specimen was a large-scale high strength steel MRF with fuse beams of carbon mild steel in the short bay. In particular, the two twin plane frames of two bays were connected by out-of-plane braces with a distance of $1 \mathrm{~m}$. In the frame plane, the column distance for the 
long bay and the short bay was $2 \mathrm{~m}$ and $1 \mathrm{~m}$, respectively. To restrict damages to the fuse beams, the primary MRF system was designed with high strength steel of a nominal yield strength of $460 \mathrm{MPa}$, and the nominal yield strength for the fuse beams was $235 \mathrm{MPa}$. During the experiment, the specimen was fixed on the ground through a rigid foundation beam. A vertical load of $1500 \mathrm{kN}$, simulating the gravity load, was first exerted from a vertical actuator and transferred to the columns, producing the nominal axial force ratio $\left(N / N_{\mathrm{y}}\right)$ of 0.15 for the middle columns, where $N$ and $N_{\mathrm{y}}$ are the axial force measured by strain readings and the nominal axial force causing yielding of the column, respectively. For a system in high seismic regions, a relatively small axial force ratio induced by the gravity load is rational [26]. This axial force could also indirectly account for the effect of the gravity load of a structure on the columns and involve the P-delta effect under seismic actions. Subsequently, to closely simulate the actual lateral loads induced by seismic actions, the triangular lateral loads (the ratio of the horizontal load at the first story to the load at the second story is $1: 2$ ) were applied on the specimen, assuming that the system would undergo the invariant triangular inertia force distribution under earthquake events. In the test, the specimen was cyclically loaded in terms of story drift. In particular, the actuator in the second story was set as the driving actuator, and the one in the first story was set as the driven actuator. During the loading process, the driving actuator was controlled in terms of displacement, and the drift of the second story measured by displacement transducers was used to govern the load cycles. This test setup was also used in a companion research work [40]. Note that although the quasi-static test could not perfectly replicate inertia forces induced by ground motions, it could still provide an 
insight into the nonlinear behavior of the system, and the test results could also be utilized as a sound base for establishing structural hysteretic models for dynamic analyses.

The story shear force versus story drift curves loaded to the controlled drift of $4 \%$ are given in Fig. 2(b). Also, the damage-control cores corresponding to the curves with the drift loaded to $1.5 \%$ beyond which the high strength steel beams in the primary system began to develop remarkable inelastic deformation, as indicated by the strain readings, are extracted and given in Fig. 2(c). In these figures, $V_{1}$ and $V_{2}$ denote the story shear force of the first story and the second story, respectively; $\theta_{1}$ and $\theta_{2}$ are the corresponding story drifts. To validate the applicability of the bilinear hysteretic model of significant post-yielding stiffness ratio for featuring the DCC, the bilinear model curves were determined and compared with the test curves. In particular, the skeleton curves up to the controlled drift of $1.5 \%$ were idealized by a bilinear estimation following FEMA 273 [10], and the initial stiffness, the equivalent yielding story drift corresponding to the yielding of fuse beams $\left(\theta_{\mathrm{y} 1}\right)$ and the post-yielding stiffness ratio $(\alpha)$ were determined and presented in Fig. 2(c). Subsequently, the drift history measured in the test and the calibrated coefficients ( $\alpha$ and $\theta_{\mathrm{y} 1}$ ) were used to determine the model curves. As can be seen, satisfactory agreement between the bilinear model curves of significant post-yielding stiffness ratios and the test curves can be observed. In general, although the realistic structural hysteretic curves are not perfectly bilinear because yielding develops progressively, the bilinear model of significant post-yielding stiffness ratio can produce satisfactory estimates for predicting the structural seismic response and at the same time retain computation efficiency as also illustrated in previous research works [33-34]. For low-to-medium rise frame systems which are 
dominated by racking deformations, the seismic response of the entire system can be reasonably evaluated considering an equivalent SDOF system of the applicable hysteretic behavior $[8,15]$. Therefore, bilinear SDOF systems with considerable post-yielding stiffness ratios are utilized to analyze the seismic response of frame systems with energy dissipation fuses considering the damage-control behavior.

\subsection{Energy demand indices}

To quantify the nonlinear behavior of the system, the post-yielding stiffness ratio $(\alpha)$ of the DCC is defined as the ratio of the post-yielding stiffness to the initial stiffness of the system. The sequence factor is defined and given by

$$
\zeta=\frac{\delta}{\delta_{y 1}}
$$

where $\delta_{\mathrm{y} 1}$ and $\delta$ are defined as the first yield displacement determined by yielding of fuses and the expected target displacement, respectively. As yielding of the primary system results in increasing of post-earthquake repair works and great loss of structural functionality, the deformation denoting the initiation of yielding in the primary system $\left(\delta_{\mathrm{y} 2}\right.$ in Fig. 1) is defined as the resilience threshold. Correspondingly, the value of $\zeta_{\mathrm{T}}$ (Fig. 1) quantifying the deformation range of a DCC can be determined.

Considering the energy balance of system below the resilience threshold, the energy factor $\gamma_{\mathrm{dc}}$ is determined using the nominal absorbed energy calculated by the covered area of the skeleton curve to the absorbed energy of the corresponding elastic system ratio with a target displacement, given by

$$
\gamma_{\mathrm{dc}} E_{\mathrm{ae}}=E_{\mathrm{a}}
$$

where $E_{\mathrm{ae}}$ and $E_{\mathrm{a}}$ are the absorbed energy of the corresponding elastic system and nominal 
absorbed energy of an inelastic system, as illustrated in Fig. 3. Therefore, the energy factor can be calculated and given by

$$
\begin{gathered}
\gamma_{\mathrm{dc}}=\frac{E_{\mathrm{a}}}{\frac{1}{2} M S_{\mathrm{v}}^{2}}=\frac{\frac{1}{2} V_{\mathrm{y} 1} \delta_{\mathrm{y} 1}+(\zeta-1) V_{\mathrm{y} 1} \delta_{\mathrm{y} 1}+\frac{1}{2}(\zeta-1)^{2} \alpha V_{\mathrm{y} 1} \delta_{\mathrm{y} 1}}{\frac{1}{2} V_{\mathrm{e}} \delta_{\mathrm{e}}}=\chi\left[2 \zeta-1+\alpha(\zeta-1)^{2}\right] \\
\chi=\left(\frac{V_{\mathrm{y} 1}}{V_{\mathrm{e}}}\right)^{2}
\end{gathered}
$$

where $M$ is the mass of the system; $S_{\mathrm{v}}$ is the spectral pseudo-velocity determined from an elastic spectral analysis of an SDOF system under a ground motion [15,17], and it can be utilized to quantify the peak response of an elastic SDOF system; $V_{\mathrm{y} 1}$ and $\delta_{\mathrm{y} 1}$ are the force and displacement corresponding to yielding of fuses, respectively; $V_{\mathrm{e}}$ and $\delta_{\mathrm{e}}$ are the maximum force, maximum displacement of corresponding elastic system, respectively; $\chi$ is defined as the damage-control factor, which is dependent on the parameters of the DCC and ground motion properties simultaneously. According to the aforementioned derivation, it can be seen that the energy factor of systems with fuses is not identical to EP systems and affected by the DCC shape.

As for the cumulative ductility for a system with fuses, it is also not identical to EP systems [29-30]. In particular, the cumulative ductility demand [34] can be solved and given by

$$
\mu_{\mathrm{a}}=\frac{E_{\mathrm{p}}}{(1-\alpha) V_{\mathrm{y} 1} \delta_{\mathrm{y} 1}}
$$

where $E_{\mathrm{p}}$ is the plastic energy dissipated by systems. For structures deforming in the range below the resilience threshold, $\mu_{\mathrm{a}}$ can be used to quantify the cumulative demand induced by ground motions with a focus on the damage-control behavior. 
It is noted that $\gamma_{\mathrm{dc}}$ determined from the classical energy balance equation proposed by Housner [15,17] is obtained based on a skeleton pushover curve of the entire system, and the elastic energy contributed by the primary system is also involved. Thus, this index also serves as a systematic parameter. Comparatively, $\mu_{\mathrm{a}}$ is a normalized quantity determined from the dissipated plastic energy corresponding to damages restricted in fuses. Considering the essence of the structural energy balance during a ground motion, it is not the total energy of an earthquake that determines the damage of a structure, but the rate at which this energy arrives and shakes the structure [41-42]. This indicates that both the peak response and the cumulative response are significant in identifying the structural damage and behavior, which can be considered by the energy factor and the cumulative ductility, respectively. In this respect, these two indices can serve as demand indices to comprehensively identify the seismic energy demand of the system, echoing the two fundamental requirements of damage-control behavior addressed in Section 2.1.

\section{Parametric study of energy demand indices}

\subsection{Development of the demand indices spectra and ground motions}

The procedure of computing the energy demand indices is given in the flowchart illustrated in Fig. 4. This procedure is similar to establishing a constant ductility spectrum $[18,25]$, in which the sequence factor and the post-yielding stiffness ratio for quantifying the DCC are used as basic parameters. During these analyses, the determination of $V_{\mathrm{y} 1}$ follows an iteration procedure. In particular, the iteration starts from assigning an initial value of $V_{\mathrm{y} 1}$ (close to $V_{\mathrm{e}}$ ) to the SDOF system and running the NL-RHA of the SDOF system. Then, the procedure is repeated with value of $V_{\mathrm{y} 1}$ decreasing gradually until the 
expected value of $\zeta$ is achieved.

To establish a parametric study, 120 ground motions records (Fig. 5) which can be regarded as representatives of different soil conditions and fault effects are used in this research. These ground motions were also used in the original SAC project [43] representing the stiff soil, soft soil and near-fault conditions, respectively. In the analyses, 50 structural periods ranging from $0.1 \mathrm{~s}$ to $5 \mathrm{~s}$ are considered and a constant damping ratio of $5 \%$ is assumed. To compare with the conventional hysteretic model, the corresponding EP systems are also incorporated into the analysis. 210000 analyses are performed considering SDOF systems with various DCC shapes, and a validated algorithm BTESPEC [16] is used to perform the analyses.

\subsection{Energy factor spectra and cumulative ductility spectra}

As representative results, mean values of $\gamma_{\mathrm{dc}}$ with different DCC parameters are plotted in Fig. 6. As can be seen, for the effect of $\zeta, \gamma_{\mathrm{dc}}$ in short period regions $(T<0.5 \mathrm{~s})$ increases with increasing $\zeta$, highlighting the phenomenon of "energy lock". This explains the classical equal-strength rule $[1,15]$ for short-period systems in the perspective of energy, implying that the peak response demand can be very large for short-period systems developing inelastic deformation. Comparatively, increasing of $\zeta$ leads to favorable decreasing of $\gamma_{\mathrm{dc}}$ in moderate and long period regions $(T>0.5 \mathrm{~s})$, indicating that the energy balance can be more readily to reach by making use of plastic deformation of fuses. This phenomenon indicates that enlargement of yield drift gaps between the primary system and fuse elements is an effective alternative to realize the damage-control behavior for moderate and long period structures, since $\gamma_{\mathrm{dc}}$ decreases significantly. For the feature of the 
post-yielding stiffness ratio, the results show that the increasing value of $\alpha$ leads to an evident decrease of $\gamma_{\mathrm{dc}}$ in short period regions while a reversed trend can be observed in longer period regions. Especially in cases where the sequence factor is significant, the amplification of $\gamma_{\mathrm{dc}}$ induced by $\alpha$ in the long period region is substantial. For instance, for systems with the period of $3 \mathrm{~s}$, there is averagely a $100 \%$ increase of $\gamma_{\mathrm{dc}}$ considering the stiff soil site of LA area ( $\zeta=5)$, when $\alpha$ increases from 0 to 0.7 (See Fig. 6(a)). In general, although the effect of the shape of DCC on the energy factor is not appreciable for systems of relatively small $\alpha$ and $\zeta$ with the period larger than $0.5 \mathrm{~s}$, the evident effect of the DCC on the energy factor can be observed for cases with significant $\alpha$ and $\zeta$. Also, the widely used energy factor spectra [19-23] derived from the Newmark and Hall spectra [1] for EP systems are also indicated in the figure (See Fig. 6(a)), and an evident difference can be observed for systems with a DCC of large $\alpha$ and $\zeta$, hence, strengthening the necessity of considering the DCC for designing and evaluating structures with fuses. Note that although only representative results are given, the tendency is the same for all ground motion ensembles. To provide a more comprehensive insight into the behavior of the system, the corresponding yield force spectra featured by normalized $V_{\mathrm{y} 1}$, determined as $C_{\mathrm{y}}=V_{\mathrm{y} 1} / W(W$ denotes the seismic weight of the SDOF system), was obtained and plotted in Fig. 6. In general, $C_{\mathrm{y}}$ decreases with increasing $\zeta$, which is expected since the sufficient development of inelastic deformation leads to the lower demand in terms of the yield strength of a system. As for the effect of $\alpha$, it produces a more evident effect on systems with large $\zeta$ in the relatively short period region. Compared with the corresponding EP system, systems of significant $\alpha$ also show the lower demand of $V_{\mathrm{y} 1}$ for structures from the short period to 
moderately long period regions $(T<1 \sim 1.5 \mathrm{~s})$.

Fig. 7 presents representative mean cumulative ductility $\left(\mu_{\mathrm{a}}\right)$ spectra considering different shapes of DCC. For EP systems $(\alpha=0), \mu_{\mathrm{a}}$ almost keeps constant irrespective of the variation of period. In contrast, for systems employing a DCC, larger $\zeta$ and $\alpha$ lead to a significant increase of $\mu_{\mathrm{a}}$, which is more drastic when the period is below $2 \mathrm{~s}$.

From these analyses results, it can also be observed that the employment of a DCC of significant $\alpha$ reduces the dependency of $\gamma_{\mathrm{dc}}$ on site conditions. Comparatively, this tendency is not remarkable for $\mu_{\mathrm{a}}$ as it shows dependency on site conditions for both EP SDOF systems and the counterparts with a DCC of significant $\alpha$. However, for different site conditions, the difference of $\mu_{\mathrm{a}}$ of systems ranging from short period to moderately long period regions $(T<1 \sim 1.5 \mathrm{~s})$ with a significant $\alpha$ and $\zeta$ becomes more remarkable. The evidence supporting this phenomenon can be extracted by comparing demand indices of different ground motion ensembles, and the typical results are plotted in Fig. 8 and Fig. 9. In particular, for a system with a DCC of significant post-yielding stiffness ratio, taking $\alpha=0.7$ as an example, the difference between energy factors under various ground motion ensembles is not as evident as that between EP systems. Comparatively, the cumulative ductility of both EP systems and the counterparts with a DCC is sensitive to site conditions, as indicated by the distinct differences of this quantity under various ground motion ensembles, and the difference will be amplified for systems with a DCC from short period to moderately long period regions. These results also reinforce the necessity to account for the dual energy demand indices considering both peak response and cumulative response simultaneously. 
For practical applications, regression analyses (see Appendix A) are performed to relate the mean energy demand indices with structural properties, which can be used to estimate the mean demand considering these site conditions. Even though the rationality of these analyses results are conditioned on the ability of the SAC ground motions to suitably represent site soil conditions, site seismicity, and fault effects, the required demand indices in practical cases can be generated from any potential ground motion using the proposed algorithm without complicated computation efforts.

\subsection{Dispersion of demand indices}

For the energy factor, the dispersion spectra composed of coefficients of variation $(\mathrm{COV})$, the ratio of the standard deviation to the mean values are generated. Representative results are shown in Fig. 10. With $\zeta$ increasing, the dispersion increases while the enhancement of the post-yielding stiffness ratio can favorably eliminate this effect. When $\alpha$ is adequately large, the COV is relatively small and almost keeps constant regardless of the variation of the period. Fig. 11 presents the representative dispersion spectra regarding the cumulative ductility. Compared with results of the energy factor, the dependency of the COV of $\mu_{\mathrm{a}}$ on the shape of DCC is more erratic, except for the cases of near-fault ground motions, and there is not an indication that the increasing of $\alpha$ produces $\mu_{\mathrm{a}}$ with reduced dispersion. These results show that the employment of a DCC may favorably eliminate the dispersion regarding the peak response as indicated by the COV of the energy factor, but the dispersion regarding the cumulative effect should be considered carefully. In this respect, although both energy factor and cumulative ductility are critical indices for quantifying the seismic demand, the energy factor serves as a more instructive index 
considering the dispersion, and the necessity of applying the dual energy demand indices is demonstrated again. Essentially, if the fuse elements in a system do have redundant energy dissipation capacity to sustain all cumulative damages, a DCC of significant post-yielding stiffness ratio is then more preferable since the uncertainty of the peak response related to primary system damages can be reduced.

\section{Dual-energy-demand-indices-based (DEB) damage-control evaluation procedure}

For low-to-medium rise frame structures dominated by racking deformations under ground motions, although the structures behave as multi-degree-of-freedom (MDOF) systems, the behavior can be evaluated with the equivalent SDOF systems considering the fundamental vibration mode reasonably [13-15], and the dynamic behavior of the SDOF system can be used for quantifying the response of the entire system. Thus, the damage-control behavior can be evaluated using the dual energy demand indices determined from the corresponding SDOF systems. Specifically, for the peak response of the system, the energy balance can be evaluated with the nominal energy demand and capacity with the focus of the energy factor, and the potential damages of the primary system can be identified. Subsequently, the cumulative effect of the system can be assessed using the cumulative ductility index. Therefore, a stepwise procedure defined as dual-energy-demand-indices-based (DEB) damage-control evaluation procedure, which can be used to evaluate and optimize a design strategy is established in this research and presented as follows:

Step 1: Calculate the elastic vibration properties of the system considering the fundamental mode (period, effective mass, the participation factor). 
Step 2: Perform a pushover analysis and develop nominal energy capacity curves with an incremental approach as proposed in [24, 44]. At its core, a system is firstly transformed to an energy-based SDOF (Fig. 12) system by developing the base shear versus the energy-based displacement $\left(u_{\mathrm{e}}\right)$ curves and the base shear versus the sequence factor curves. In particular, the invariant lateral load distribution is first assumed and given as

$$
\mathbf{S}=\mathbf{m} \varphi_{1}
$$

where $\mathbf{S}$ and $\mathbf{m}$ are the lateral load distribution vector and the mass matrix, respectively; $\boldsymbol{\varphi}_{\mathbf{1}}$ is the modal vector of the fundamental mode. As mentioned before, for low-to-medium rise MDOF systems dominated by racking deformations under ground motions, applying the equivalent SDOF system considering the fundamental mode can still lead to reasonable estimates and the rationale was validated by relevant research works $[15,17,44]$. Recognizing that the external work done by the lateral loads is identical to the absorbed energy of the system, which is governed by the energy equilibrium principle, the energy-based displacement $\left(u_{\mathrm{e}}\right)$ is quantified, which can be interpreted graphically by the covered area of the pushover curve, as given in Fig. 12(b). In the elastic domain, $u_{\mathrm{e}}$ can be directly determined with the base shear $V_{\mathrm{b}}[24,44]$ considering the linear behavior of the system. More generally, for both the elastic and the inelastic domain, since the energy equilibrium principle always holds, the work done by $V_{\mathrm{b}}$ in a differential displacement $\delta u_{\mathrm{e}}$ is necessarily equal to the work done by the lateral force in the correspondingly. Then, the nominal energy capacity versus sequence factor curve can be constructed. This step can be accomplished by Eq. (7) (12).

$$
\delta W^{m}=\frac{1}{2}\left(\mathbf{S}^{m-1}+\mathbf{S}^{m}\right) \cdot\left(\mathbf{U}^{m}-\mathbf{U}^{m-1}\right)
$$




$$
\begin{gathered}
\delta u_{e}^{m}=\frac{\delta W^{m}}{\frac{1}{2}\left(V_{b}^{m}+V_{b}^{m-1}\right)} \\
V_{b}^{m}=\mathbf{S}^{m} \cdot \mathbf{1} \\
W^{m}=W^{m-1}+\delta W^{m} \\
u_{\mathrm{e}}^{m}=u_{\mathrm{e}}^{m-1}+\delta u_{\mathrm{e}}^{m} \\
\zeta^{\mathrm{m}}=\frac{u_{\mathrm{e}}^{m}}{u_{\mathrm{ey}}}
\end{gathered}
$$

where $\mathbf{U}$ is the lateral displacement vector, corresponding to the displacement profile of stories; $\delta W^{m}$ is the incremental external work identical to the absorbed energy of the system at the $m$ th step; $\delta u_{\mathrm{e}}{ }^{m}$ is the incremental energy-based displacement at $m$ th step; $u_{\mathrm{ey}}$ is the equivalent energy-based yield displacement corresponding to inception of yielding of fuses, which can be accomplished by applying the bilinear idealization following the FEMA 273 [10] based on the capacity curves to the resilience threshold (Fig. 12(c)). Note that after the construction of the nominal energy capacity curves, the resilience threshold $\left(\zeta_{\mathrm{T}}\right)$ should be identified (Fig. 12(c)).

Step 3: Confirm the shape of DCC based on the base shear versus energy-based displacement curves determined from Step 2. Again, for the idealized curve, the quantified yield force and the yield displacement are equivalent features since the actual system will yield progressively, but this simplification can produce results with satisfactory accuracy and computation efficiency for quantifying structural seismic response $[8,34]$. Then, the confirmed base shear corresponding to the yielding of fuses can be substituted into the $V_{\mathrm{y} 1}$ of the equivalent SDOF system.

Step 4: After obtaining the parameters of the DCC $\left(\alpha, \zeta, \zeta_{\mathrm{T}}\right)$, compute the energy demand 
indices following the framework in Fig. 4. It is noted that during analyses of identifying the demand indices, various values of $\zeta$ should be employed as it is an essential feature for constructing the demand curves (Step 5). Comparatively, as a quantity identifying the resilience threshold, $\zeta_{\mathrm{T}}$ denotes the capacity of the system, which can be utilized to make the damage-control behavior check (Step 6). In this step, potential ground motions should be identified first, and the regressed equation (Eq. (A.1) (A.3)) in Appendix A can also be used to estimate the mean values for systems with damping ratio of $5 \%$.

Step 5: Develop the nominal energy demand curves associated with the sequence factor considering the index of the energy factor and the elastic spectral pseudo-velocity $\left(S_{\mathrm{v}}\right)$ of ground motions (Fig. 13). In particular, the demand curve can be generated by plotting the value of $E_{\mathrm{a}}$ determined by Eq. (2) against $\zeta$.

Step 6: Make the damage-control behavior check (Fig. 13). When an intersection point of the demand curve (Step 5) and the capacity curve (Step 2) is obtained below the defined resilience threshold, the nominal energy balance can be achieved, indicating that the primary system will stay damage-free, and the procedure can be conducted considering the next step. Otherwise, the inception of damages in the primary systems is expected, implying that the adjustment of the DCC is needed, which can be achieved by modifying the structural design strategy such as strengthening the primary system or weakening the fuses.

Step 7: Calculate the plastic energy with the extracted cumulative ductility demand index solved in Step 4 and Eq. (5), based on the premise that the peak response of the system is below the resilience threshold (controlled by Step 6). Therefore, the plastic energy demand 
of the entire system is determined. Note that the intersection point extracted in Step 6 should be used to quantify the corresponding sequence factor, based on which the cumulative ductility is computed for each ground motion.

Step 8: Distribute the plastic energy in stories by applying a refined energy profile method $[26,31]$. Specifically, since only the fundamental vibration mode is considered, the plastic energy absorbed by a story to the total dissipated plastic energy ratio under a ground motion is assumed to be identical to that when the system is pushed to the peak deformation under the lateral loads of Eq. (6), and the absorbed energy at each story is calculated considering the moment-curvature response of all members under the pushover analysis. Since the previous steps with the energy factor are able to quantify the peak response of the system by the intersection point of the demand curve and the capacity curve [24], the energy profile can be determined associated with the pushover database, as illustrated in Fig. 14. Again, it is necessary to point out that the applicability of this simplification is limited to low-to-medium rise structures in which the lateral response is dominated by racking deformations with a minimum effect induced by higher vibration modes. This simplification would lead to biased estimates of the energy profile if the higher vibration modes have a significant effect on the structural seismic response of the structure. Step 9: Check the cumulative effect in each story associated with the available data of fuse elements energy dissipation capacity database. Even though the available information on the energy dissipation capacity of fuse elements requires specialized investigations [28], the demand has been clarified by the plastic energy dissipation demand determined by step 8. 
Therefore, by performing the DEB procedure, both the peak demand and cumulative demand of the system are considered. The framework of DEB procedure is illustrated in Fig. 15. Since the two energy demand indices can be implemented in a stepwise procedure with the focus on the peak response and the cumulative response, respectively, the efficiency of an energy-based procedure is enhanced with more flexibility (i.e. In cases where the cumulative effect is not significant, the energy factor will suffice for the evaluation.)

\section{Application of the energy demand indices in damage-control evaluation}

To illustrate the application and advantages of the DEB procedure, two 6-story damage-control steel MRF systems with fuse beams, which can be regarded as practical applications of the experimental specimen in Section 2 are designed. The member section information about the two systems is plotted in Fig. 16. The fuse beams are arranged in the external bays. The plastic flexural strengths of the fuse beams are also shown in the figure, and the reduction of strength of fuse beams can be realized by applying low yield point steel [45-47] or reduced sections. For frame A, the primary system is designed with high strength steel [48-50] of a yield strength of $460 \mathrm{MPa}$. For frame $\mathrm{B}$, the beams of the primary system are assumed to have a yield strength of $390 \mathrm{MPa}$ while the columns are designed with high strength steel of a yield strength of $460 \mathrm{MPa}$. The structures are preliminarily designed according to the Chinese seismic code [51]. A dead load of 4.8 $\mathrm{kN} / \mathrm{m}^{2}$ and a live load of $2 \mathrm{kN} / \mathrm{m}^{2}$ are assumed. The basic acceleration is assumed to be 0.4 g. The systems are modeled with ABAQUS using B21 elements. It should be noted that a damping ratio ranging from $2 \%$ to $5 \%$ is generally used for design of steel structures, and a 
larger value can be used to rationally account for the damping effect of non-structural elements. Thus, the Rayleigh damping of $5 \%$ is assumed for the first two vibration modes in this study, which is also used in previous research works [15, 22-23, 26, 33]. However, as the core of the DEB procedure lies in applying equivalent SDOF systems to quantifying the dual-energy-based-demand indices of structural systems, the rationale of the procedure does not depend on the value of damping ratio used in the prototype structures, and engineers could use various damping ratios in practical cases. The bilinear behavior of $2 \%$ hardening ratio for all members is assumed. For the purpose of demonstration, ground motions considering the stiff soil of Los Angeles (LA01 LA20) are assumed as potential earthquakes.

Step 1: The vibration properties considering the fundamental vibration mode of the prototype structures are calculated and the results are given in Table 1.

Step 2 3: After the fundamental mode pushover analysis considering the gravity load, the base shear-sequence factor curves and the nominal energy capacity-sequence factor curves are developed. For the prototype structures, the resilience threshold is considered as the point corresponding to the initiation of yielding in the primary system, which can also be identified according to the pushover database. Therefore, the shape of the DCC of frame $\mathrm{A}$ is confirmed with $\alpha=0.60$ and $\zeta_{\mathrm{T}}=3.6$, and the counterparts for frame $\mathrm{B}$ is $\alpha=0.61$ and $\zeta_{\mathrm{T}}=3.1$.

Step 4: The corresponding $\gamma_{\mathrm{dc}}$ and $\mu_{\mathrm{a}}$ considering the fundamental vibration mode of the two systems are plotted against the sequence factor $(\zeta)$ as shown in Fig. 17 and Fig. 18, respectively. The results determined by the regression equations (Eq. (A.1) (A.3)) in 
Appendix A are also identified in the curves, demonstrating the satisfactory accuracy of these equations for predicting the mean value of $\gamma_{\mathrm{dc}}$ and $\mu_{\mathrm{a}}$. In addition, to clarify the effect of DCC, EP systems having the identical period to the systems with a DCC are also analyzed and presented in the figures. The evident difference induced by different models validates the necessity of an explicit consideration of the DCC in an energy-based procedure.

Step 5 6: The damage-control check is performed based on the identification of energy balance with the nominal capacity curves and the demand curves, as illustrated in Fig. 19(a) and Fig. 19(b) for frame A and frame B, respectively. According to the results, it is predicted that for frame A subject to the ground motion ensemble, there would be inelastic deformations in the primary system in cases of LA09, LA10, LA11, LA13, LA14, LA15, LA16, LA17, LA18, and LA20, since intersection points of the nominal capacity curve and the demand curves are above the resilience threshold. Likewise, it is expected that in cases of LA03, LA09, LA11, LA14, LA15, LA16, LA17, LA18, and LA20, frame B reaches the stage in which the primary system starts to yield. To validate the accuracy of the prediction, the results of the two frames in terms of plastic energy dissipated by the primary system during ground motions computed by NL-RHA are also presented in Fig. 19(a) and Fig. 19(b). The satisfactory accuracy of the prediction considering the plastification of the primary system subject to ground motions demonstrates the effectiveness of the procedure and the demand indices.

Step 7 8: For cases in which the structure can sustain damages in the fuse beams, the results of the cumulative ductility of frame A and frame B are extracted from the results 
shown in Fig. 18(a) and Fig. 18(b) based on the sequence factor denoted by the intersection points in Fig. 19. Subsequently, the plastic energy demand can be confirmed based on the equivalent SDOF system following Eq. (5). Then, the energy profiles can be determined associated with the pushover database. For frame A and frame B, the results considering the plastic energy distribution determined by the DEB procedure and NL-RHA are illustrated in Fig. 20(a) and Fig. 20(b), respectively, and the satisfactory accuracy of the DEB procedure for predicting the plastic energy distribution is validated.

Therefore, by performing the DEB procedure with the energy factor and the cumulative ductility demand, the damage-control behavior of a low-to-medium rise frame structure with energy dissipation fuses can be evaluated considering both peak response and cumulative effect, which is significant for identification of damages in both fuses and the primary system.

\section{Conclusions}

The energy factor and the cumulative ductility are used as the basic demand indices to establish an evaluation procedure considering damage-control frame structures with energy dissipation fuses. This paper firstly investigates the energy demand indices of the systems equipped with energy dissipation fuses below the resilience threshold based on a parametric study of single-degree-of-freedom (SDOF) systems, considering both the peak response and the cumulative response. Based on these indices, a stepwise energy-balance-concept-based evaluation procedure defined as dual-energy-demand-indices-based (DEB) procedure is proposed. The main findings of 
this research are listed as follows:

1. Both the energy factor and the cumulative ductility of systems with fuses are significantly different from elasto-plastic (EP) systems or bilinear systems of negligible post-yielding stiffness ratio. These indices are dependent on damage-control core shapes, structural period, and ground motion properties. These indices can be calculated following the procedure using a validated algorithm with provided ground motions.

2. The results of the parametric study indicate that the employment of a damage-control core of considerable post-yielding stiffness ratio can eliminate the dependency of the energy factor on ground motions properties and reduce the dispersion denoting the peak response demand, but it this tendency is not shown by the cumulative ductility, and the corresponding dispersion under ground motions is erratic. These phenomena reinforce the necessity to account for the dual energy demand indices in an energy-based procedure.

3. By following the DEB procedure, the peak response demand, cumulative energy demand, and the plastic energy distribution of systems below the resilience threshold under the expected ground motions can be evaluated accurately, without the necessity of performing a nonlinear response history analysis of the entire structure. In addition, the efficiency of an energy-based procedure is also enhanced with more flexibility considering the dual indices separately in a stepwise procedure. However, since the rationale of the DEB procedure lies in the accuracy of utilizing the equivalent single-degree-of-freedom (SDOF) system of the fundamental vibration 
mode to represent the dynamic behavior of the entire structure, the procedure only applies to low-to-medium rise structures in which the lateral response is dominated by racking deformations (e.g. frame structures) and the effect of higher vibration modes can be neglected.

The complete validation of the procedure still requires the energy dissipation capacity quantification, especially the ability of fuse elements to sustain the cumulative damages. Currently, research works considering the database establishment of various fuse elements base on experimental studies are in progress. In addition, the enhancement of the approach considering the effect of higher modes is also ongoing, which can provide a more comprehensive insight into the DEB procedure for engineering applications.

\section{Appendix A}

To quantify the effect of the DCC on the mean energy demand indices and to provide references for practical applications, regression analyses are performed. Two sets of regression relationships are developed: (1) the relationship of the energy factor and the parameters of the DCC, and (2) the relationship of the cumulative ductility and the parameters of the DCC. These relationships can be used to evaluate the energy demand indices for systems with fuses below the resilience threshold reasonably.

Nonlinear regression analyses are conducted to describe the mean value of $\gamma_{\mathrm{dc}}$. The form of the regression equations is given as

$$
\gamma_{\mathrm{dc}}=\frac{2 \zeta-1+\alpha(\zeta-1)^{2}}{\left[\left(\frac{\zeta-1}{a+\frac{b}{\sqrt{T}}+c e^{-T}}\right)^{\frac{1}{d}}+1\right]^{2}}
$$


where $a, b, c$ and $d$ are the regression coefficients; $T$ is the period. The form of regression equation is based on the energy balance of systems and a previous study proposed by Hatzigeorgiou [6] focusing on the relationship between the ductility and the strength reduction factor. Since the energy factor could also be expressed by the ductility and strength reduction factor [15], they are applied to construct the regressed equation. Table A1 shows the regression coefficients developed based on NL-RHA of SDOF systems with various nonlinear parameters. Comparisons between the mean energy factors and regression results are given in Fig. A1. As can be seen, the regression model provides a reasonably good fit to the mean spectra.

To quantify the relationship of mean cumulative ductility with the parameters of the DCC, nonlinear regression analyses are performed. As the parametric study addressed in Section 3 indicates that $\mu_{\mathrm{a}}$ is also dependent on the period, it is considered in the regression. In this research, the regression equation is developed by modifying the results proposed by Farrow and Kurama [3] and given by

$$
\begin{gathered}
\mu_{\mathrm{a}}=f(\zeta-1)^{K} \\
K=\frac{T^{g}}{T^{g}+1}+\frac{h}{T}
\end{gathered}
$$

where $f, g$ and $h$ are the regression coefficients. Table A2 shows the regression coefficients corresponding to different post-yielding stiffness ratios. Fig. A2 compares the results calculated by the regression equations and the mean demands determined from NL-RHA of SDOF systems. 


\section{Acknowledgments}

This research is partly supported by the National Science Foundation of China (Grant No. 51038008 and No.51578403) and the Fundamental Research Funding of China Ministry of Science and Technology (Grant No. SLDRCE14-A-03). Any opinions, findings, and conclusions presented in this paper are those of the authors and do not necessarily reflect the views of the sponsors. Authors also appreciate the constructive comments from Prof. Yiyi Chen at Tongji University, China.

\section{References}

[1] Newmark NM, Hall WJ. Earthquake spectra and design, monograph. Oakland (CA): Earthquake Engineering Research Institute (EERI); 1982.

[2] Miranda E. Site-dependent strength reduction factors. J Struct Eng ASCE 1993; 119(12): 3503-3519.

[3] Farrow KT, Kurama YC. SDOF demand index relationships for performance-based seismic design. Earthq Spectra 2003; 19(4): 799-838.

[4] Jalali RS, Trifunac MD. A note on strength reduction factors for design of structures near earthquake faults. Soil Dyn Earthq Eng 2008; 28(3): 212-222.

[5] Hatzigeorgiou GD, Beskos DE. Inelastic displacement ratios for SDOF structures subjected to repeated earthquakes. Eng Struct 2009; 31(11): 2744-2755.

[6] Hatzigeorgiou GD. Ductility demand spectra for multiple near- and far-fault earthquakes. Soil Dyn Earthq Eng 2010; 30(4): 170-183.

[7] Hatzigeorgiou GD, Papagiannopoulos GA, Beskos DE. Evaluation of maximum seismic displacements of SDOF systems from their residual deformation. Engineering Structures 2011; 33(12): 3422-3431. 
[8] Chopra AK, Chintanapakdee C. Inelastic deformation ratios for design and evaluation of structures: Single-degree-of-freedom bilinear systems. J Struct Eng ASCE 2004; 130(9): 1309-1319.

[9] CEN. EuroCode 8: Design Provisions for Earthquake Resistance-Part 1: General Rules, Seismic Actions and Rules for Buildings. European Committee for Standardization: Brussels, 2004.

[10] Federal Emergency Management Agency. NEHERP provisions for the rehabilitation of buildings. FEMA No.273 (Guidelines) and 274 (Commentary), Washington, DC, 1997.

[11] Fajfar P. Capacity spectrum method based on inelastic demand spectra. Earthq Eng Struct Dyn 1999; 28(9): 979-993.

[12] Chopra AK, Goel RK, Chintanapakdee C. Evaluation of a modified MPA procedure assuming higher modes as elastic to estimate seismic demands. Earthq Spectra 2004; 20(3): 757-778.

[13] Guo JWW, Christopoulos C. Performance spectra based method for the seismic design of structures equipped with passive supplemental damping systems. Earthquake Eng Struc Dyn 2013; 42(6): 935-952.

[14] Guo JWW, Christopoulos C. A procedure for generating performance spectra for structures equipped with passive supplemental dampers. Earthquake Eng Struc Dyn 2013; 42(9): 1321-1338.

[15] Leelataviwat S, Saewon W, Goel SC. Application of energy balance concept in seismic evaluation of structures. J Struct Eng 2009; 135(2): 113-121.

[16] Ke K, Chuan GH, Ke SZ. Seismic energy factor of self-centering systems subjected to near-fault earthquake ground motions. Soil Dyn Earthq Eng 2016; 84: 169-73.

[17] Housner GW. Limit design of structures to resist earthquakes. In: Proceedings of the 
first world conference on earthquake engineering. 1959.

[18] Zhai C, Ji D, Wen W, Lei W, Xie L. Constant ductility energy factors for the near-fault pulse-like ground motions. J Earthq Eng 2016; 1-16.

[19] Sahoo DR, Chao S. Performance-based plastic design method for buckling-restrained braced frames. Eng Struct 2010; 32(9): 2950-2958.

[20] Pekcan G, Itani A M, Linke C. Enhancing seismic resilience using truss girder frame systems with supplemental devices. J Constr Steel Res 2014; 94: 23-32.

[21] Pekcan G, Linke C, Itani A. Damage avoidance design of special truss moment frames with energy dissipating devices. J Constr Steel Res 2009; 65(6): 1374-1384.

[22] Heidari A, Gharehbaghi S. Seismic performance improvement of special truss moment frames using damage and energy concepts. Earthq Eng Struct Dyn 2015; 44(7): 1055-1073.

[23] Kharmale SB, Ghosh S. Performance-based plastic design of steel plate shear walls. J Constr Steel Res 2013; 90: 85-97.

[24] Jiang Y, Li G, Yang D. A modified approach of energy balance concept based multimode pushover analysis to estimate seismic demands for buildings. Eng Struct 2010;32(5): 1272-1283.

[25] Choi H, Kim J. Energy-based seismic design of buckling-restrained braced frames using hysteretic energy spectrum. Eng Struct 2006; 28(2): 304-311.

[26] Chou CC, Uang CM. A procedure for evaluating seismic energy demand of framed structures. Earthq Eng Struct Dyn 2003; 32(2): 229-244.

[27] Benavent-Climent A, Zahran R. An energy-based procedure for the assessment of seismic capacity of existing frames: Application to RC wide beam systems in Spain. Soil Dyn Earthq Eng 2010; 30(5): 354-367.

[28] Surahman A. Earthquake-resistant structural design through energy demand and 
capacity. Earthq Eng Struct Dyn 2007; 36(14): 2099-2117.

[29] Teran-Gilmore A, Simon-Velazquez R. Use of cumulative ductility spectra within a deformation-control format for seismic design of ductile structures subjected to long duration motions. J Earthq Eng 2008; 12(1): 136-151.

[30] Teran-Gilmore A, Bahena-Arredondo N. Cumulative ductility spectra for seismic design of ductile structures subjected to long duration motions: Concept and theoretical background. J Earthq Eng 2008; 12(1): 152-172.

[31] Habibi A, Chan RWK, Albermani F. Energy-based design method for seismic retrofitting with passive energy dissipation systems. Eng Struct 2013; 46: 77-86.

[32] Manfredi G. Evaluation of seismic energy demand. Earthq Eng Struct Dyn 2001; 30(4): 485-499.

[33] Vargas R, Bruneau M. Experimental response of buildings designed with metallic structural fuses. II. J Struct Eng 2009; 135(4): 394-403.

[34] Nakashima M, Saburi K, Tsuji B. Energy input and dissipation behaviour of structures with hysteretic dampers. Earthq Eng Struct Dyn 1996; 25(5): 483-496.

[35] Connor JJ, Wada A, Iwata M, Huang YH. Damage-controlled structures .1. Preliminary design methodology for seismically active regions. J Struct Eng 1997; 123(4): 423-431.

[36] Shen Y, Christopoulos C, Mansour N, Tremblay R. Seismic design and performance of steel moment-resisting frames with nonlinear replaceable links. J Struct Eng 2011; 137(10): 1107-1117.

[37] Dougka G, Dimakogianni D, Vayas I. Innovative energy dissipation systems (FUSEIS 1-1) - Experimental analysis. J Constr Steel Res 2014; 96: 69-80.

[38] Calado L, Proença JM, Espinha M, Castiglioni CA. Hysteretic behaviour of dissipative bolted fuses for earthquake resistant steel frames. J Constr Steel Res 2013; 
85: 151-162.

[39] Mansour N, Christopoulos C, Tremblay R. Experimental validation of replaceable shear links for eccentrically braced steel frames. J Struct Eng 2011; 137(10): $1141-1152$.

[40] Ke K, Chen YY. Seismic performance of MRFs with high strength steel main frames and EDBs. Journal of Constructional Steel Research 2016; 126: 214-228.

[41] Trifunac MD. Energy of strong motion at earthquake source. Soil Dyn Earthq Eng 2008; 28(1): 1-6.

[42] Trifunac MD. Power design method. In: Proceedings of earthquake engineering in the 21 st century to mark 40th anniversary of IZIIS-Skopje, key note lecture, 28 August-1 September 2005, Macedonia: Skopje and Ohrid, 2005.

[43] Shome N, Cornell CA, Bazzurro P, et al. Earthquakes, records, and nonlinear responses. Earthq Spectra 1998; 14(3): 469-500.

[44] Hernandez-Montes E, Kwon OS, Aschheim MA. An energy-based formulation for first- and multiple-mode nonlinear static (pushover) analyses. J Earthq Eng 2004; 8(1): 69-88.

[45] Chen S, Jhang C. Cyclic behavior of low yield point steel shear walls. Thin-Walled Struct 2006; 44(7): 730-738.

[46] Atlayan O, Charney FA. Hybrid buckling-restrained braced frames. J Constr Steel Res 2014; 96(0): 95-105.

[47] Charney FA, Atlayan O. Hybrid moment-resisting steel frames. Eng J 2011; 48(3): 169-182.

[48] Sause R, Fahnestock L. Strength and ductility of HPS-100W I-girders in negative flexure. J Bridge Eng 2001; 6(5): 316-323.

[49] Green PS, Sause R, Ricles JM. Strength and ductility of HPS flexural members. J 
Constr Steel Res 2002; 58(5-8): 907-941.

[50] Bjorhovde R. Development and use of high performance steel. J Constr Steel Res 2004; 60(3-5): 393-400.

[51] CMC. Code for Seismic Design of Buildings (GB 50011-2010). China Ministry of Construction: Beijing, 2010. 

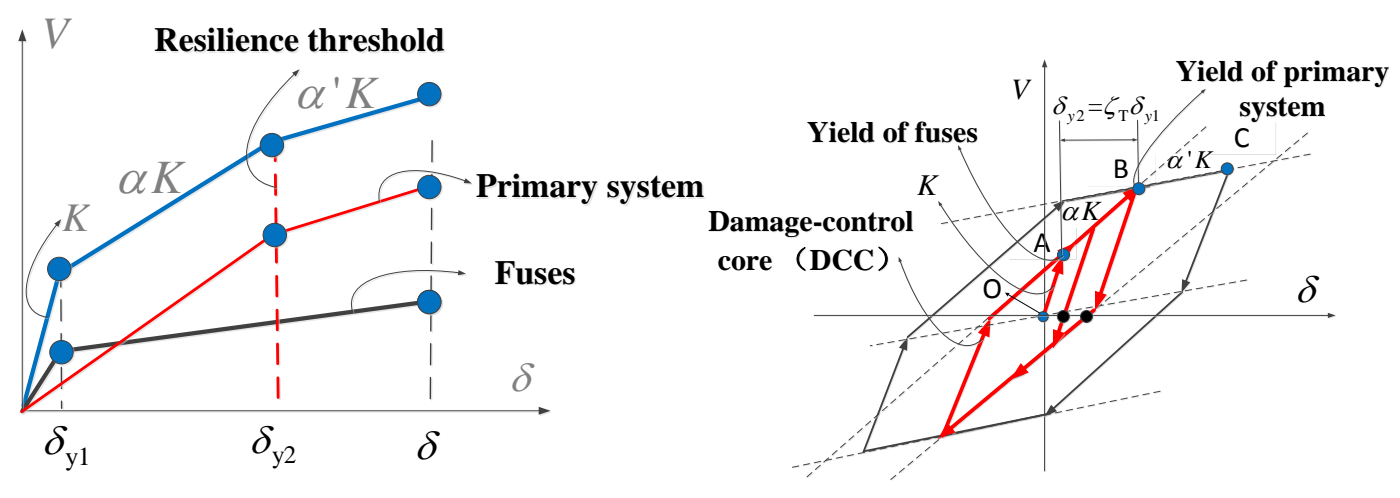

Fig. 1. Behavior of systems with fuses.

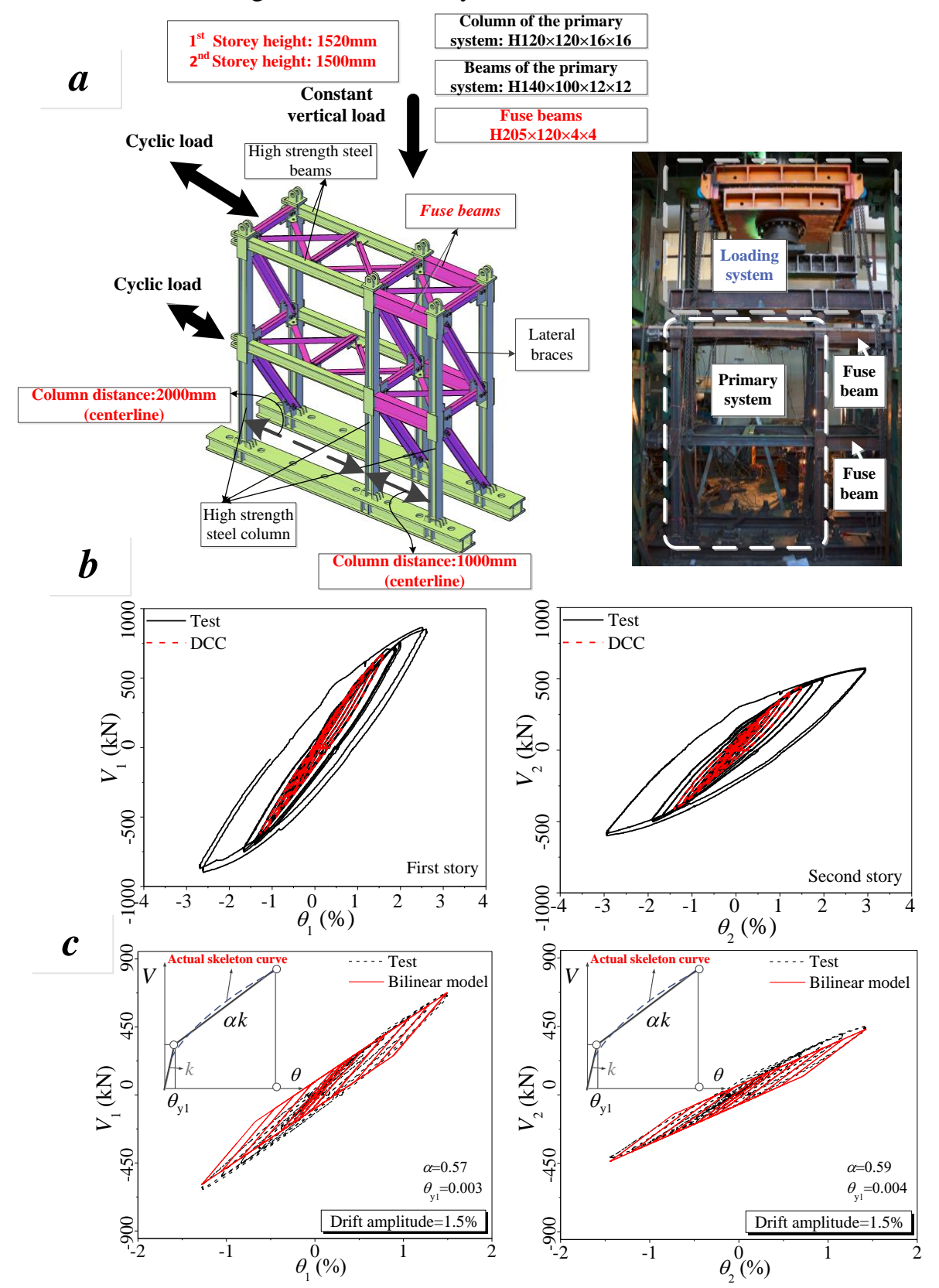

Fig. 2. Experimental validation of damage-control core (DCC): (a) Illustration of the experimental program, (b) Test curves loaded to 4\% with the DCC, (c) DCC and model validation. 


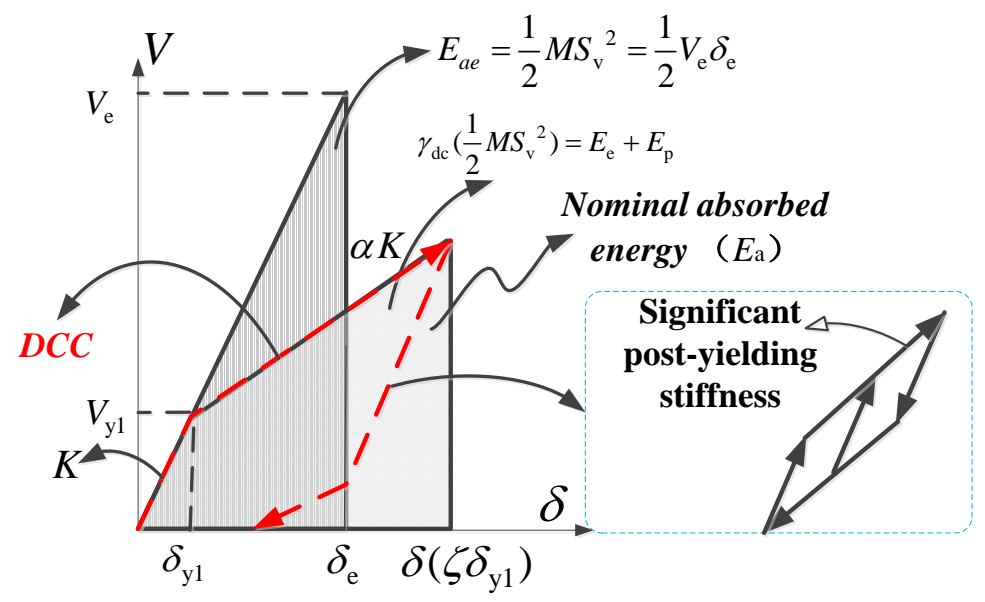

Fig. 3. Energy balance and the energy factor of systems with fuses below the resilience threshold.

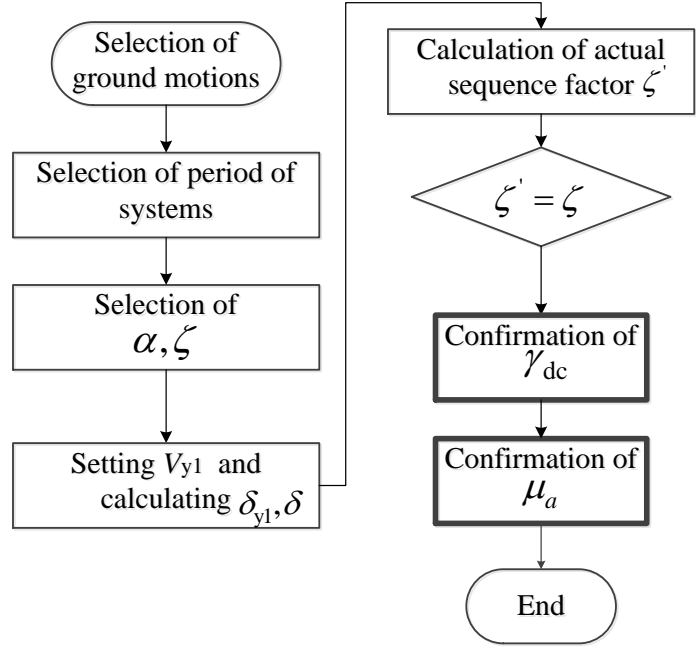

Fig. 4. Framework for computation of the energy demand indices. 

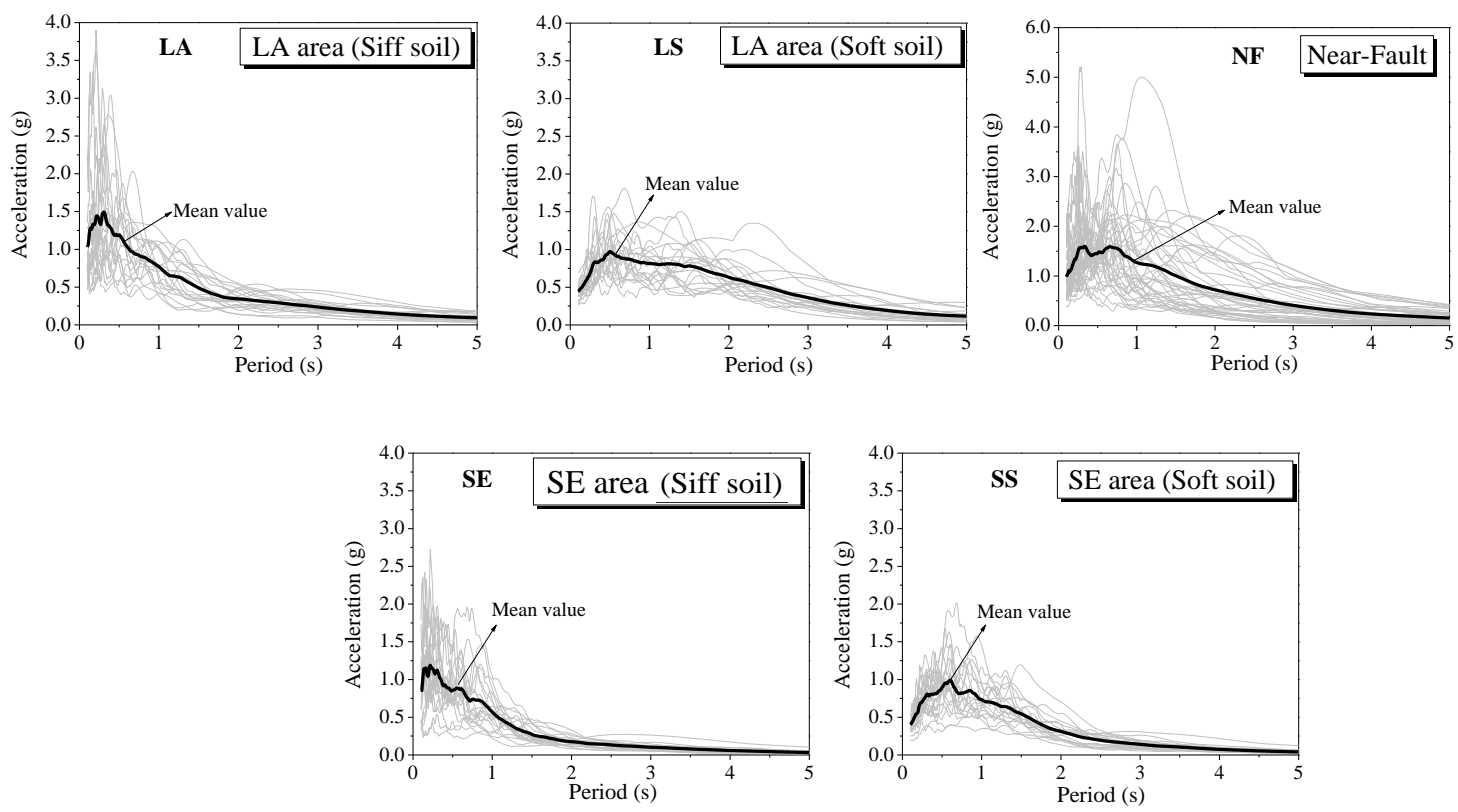

Fig. 5. Acceleration spectra of ground motions (5\% damping). 

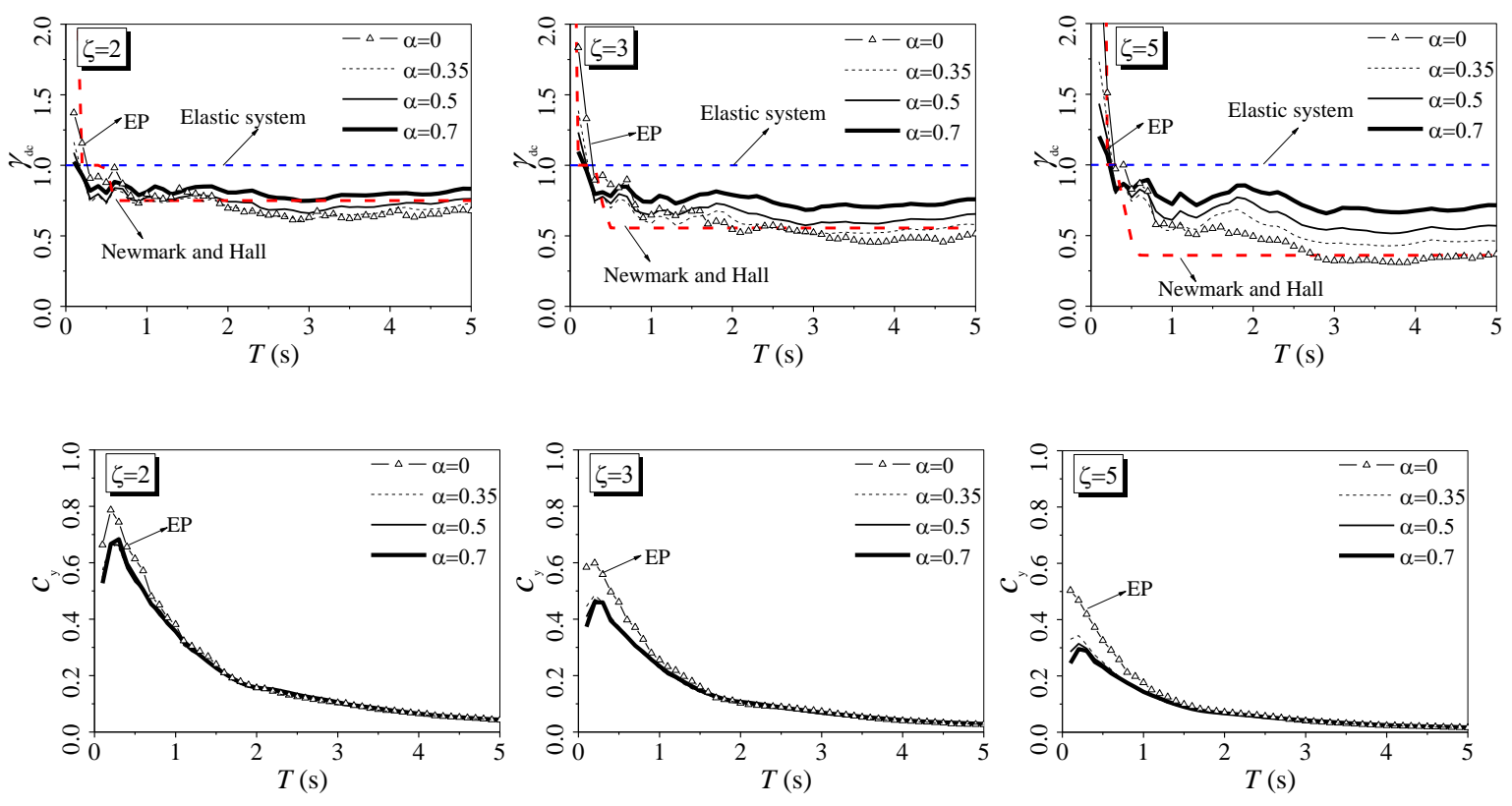

(a)
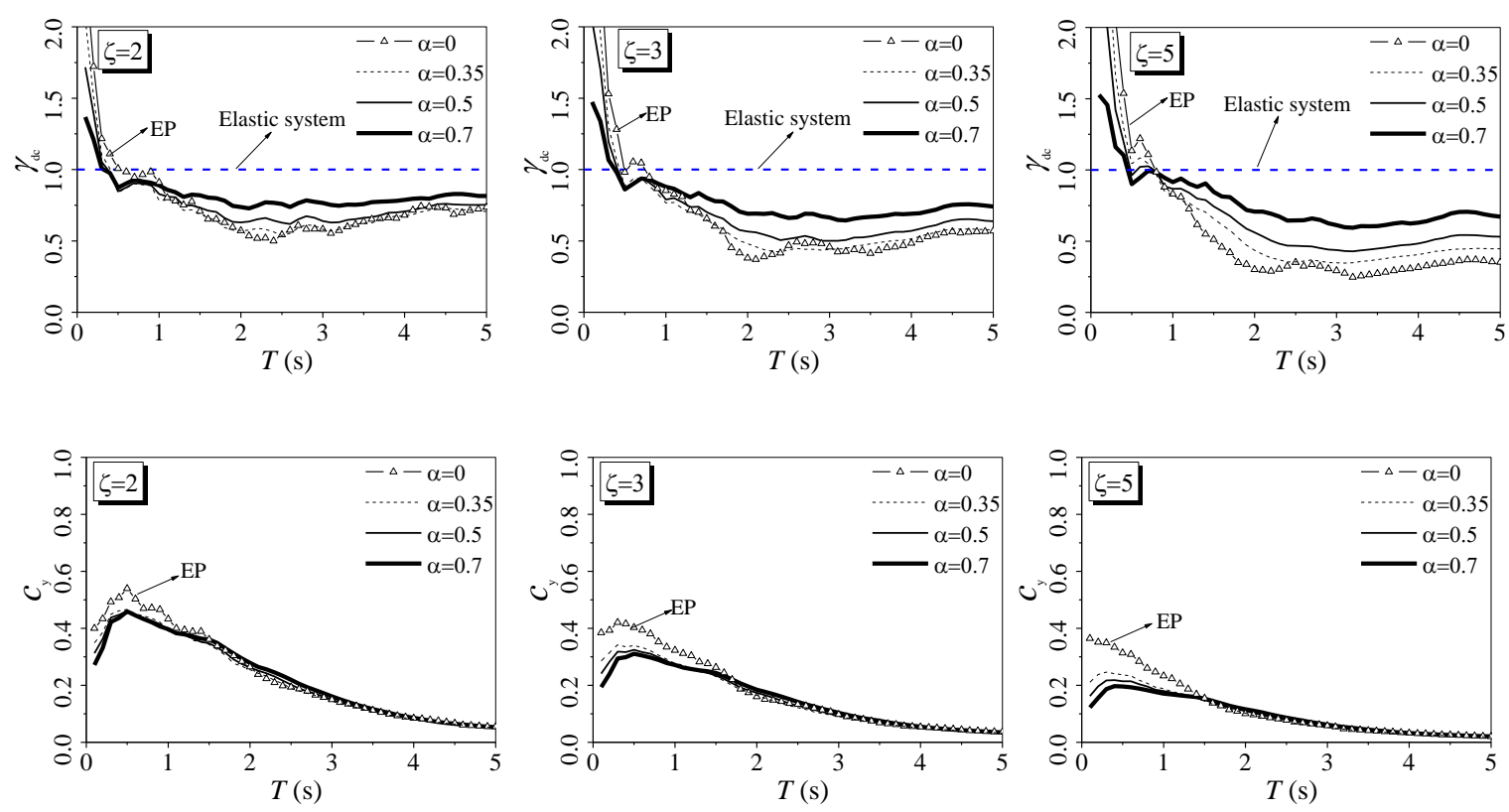

(b) 

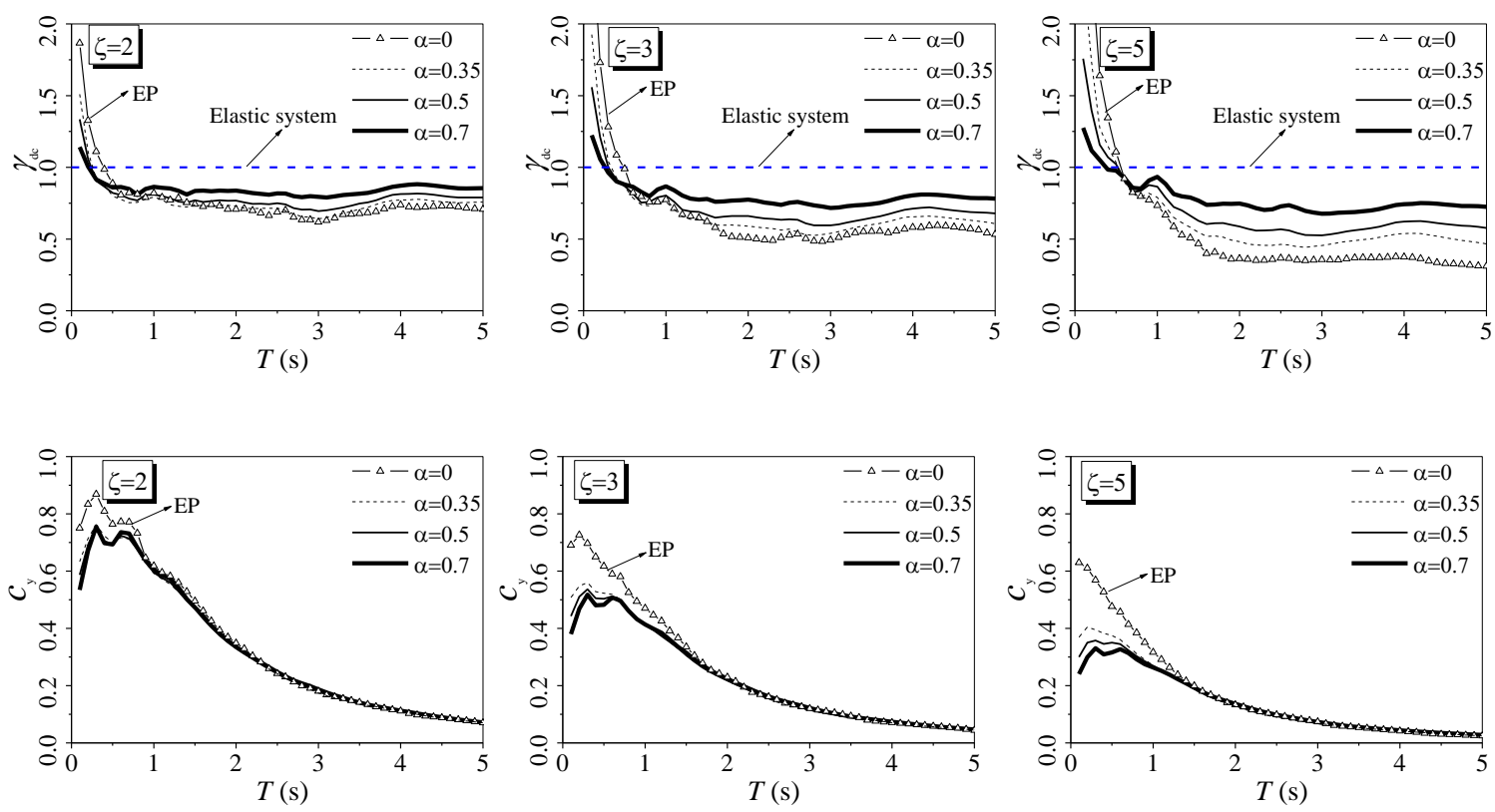

(c)

Fig. 6. Representative mean energy factor spectra and mean normalized yield force spectra: (a) Stiff soil (LA area), (b) Soft soil (LA area), (c) Near-fault ground motions. 

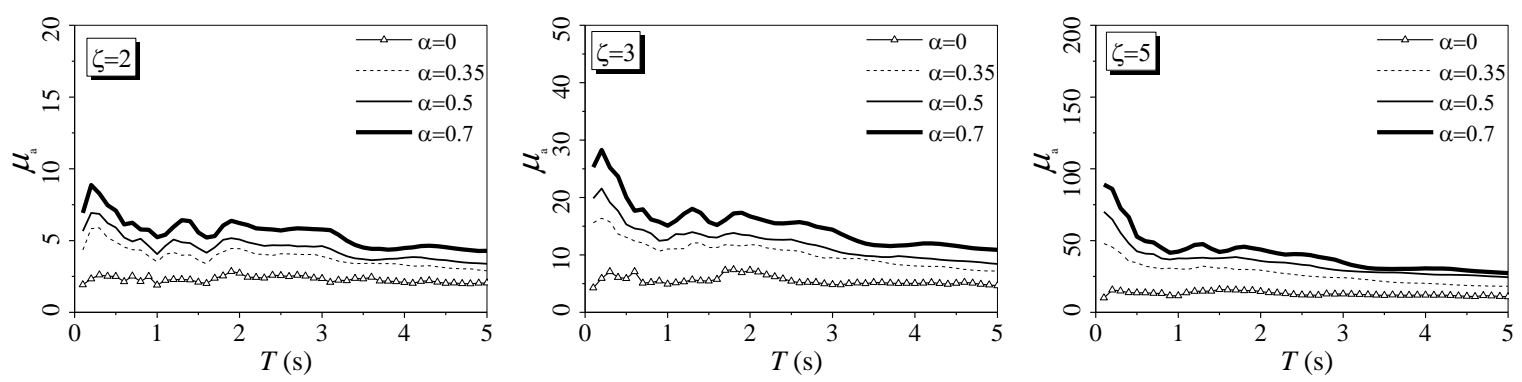

(a)
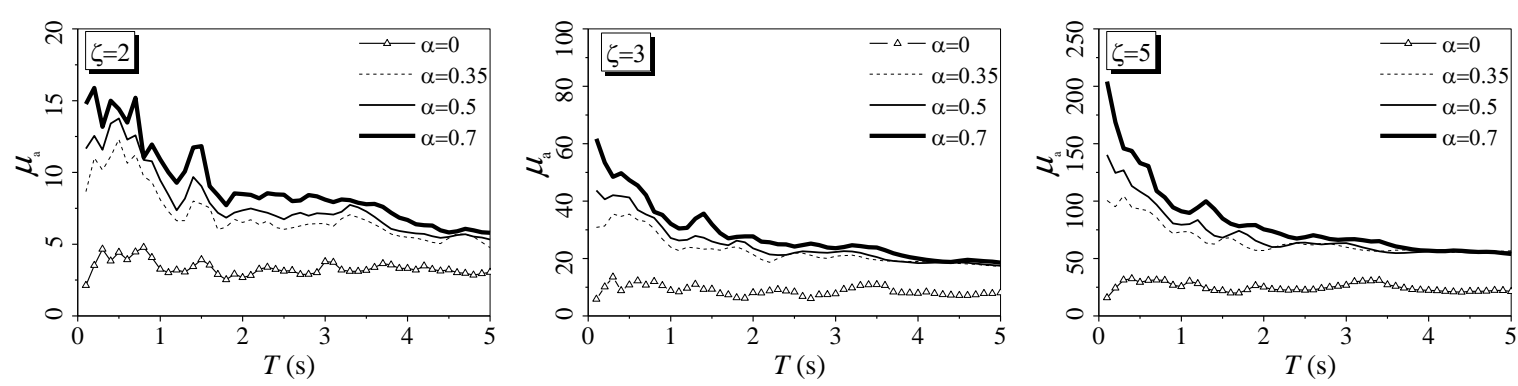

(b)
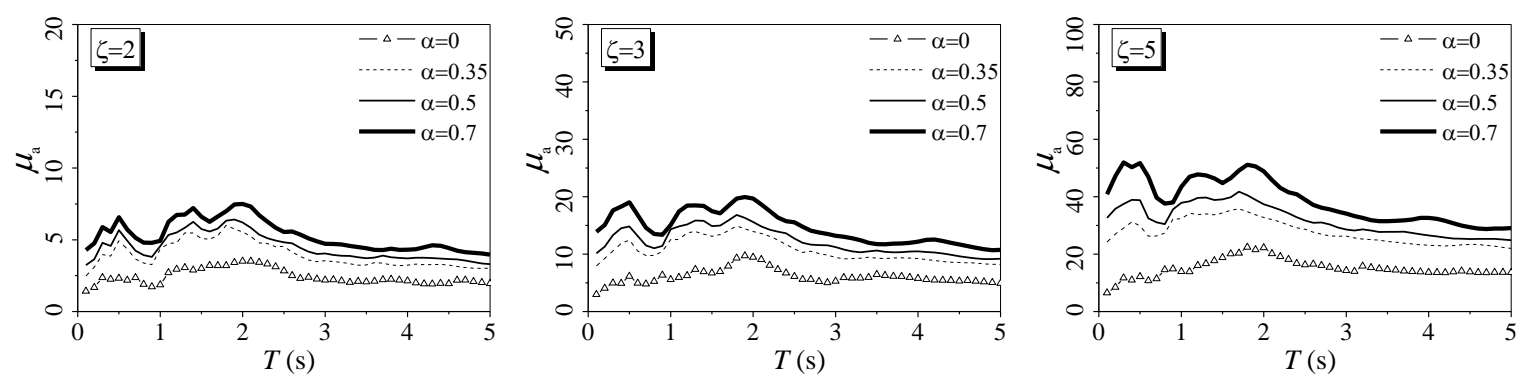

(c)
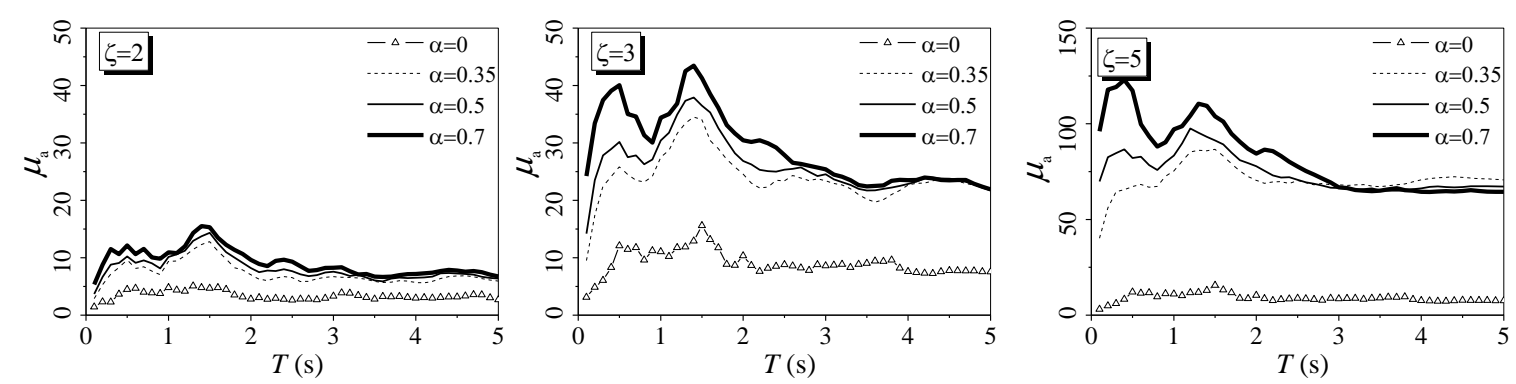

(d)

Fig. 7. Representative cumulative ductility demand: (a) Stiff soil (LA area), (b) Soft soil (LA area), (c) Stiff soil (SE area), (d) Soft soil (SE area). 

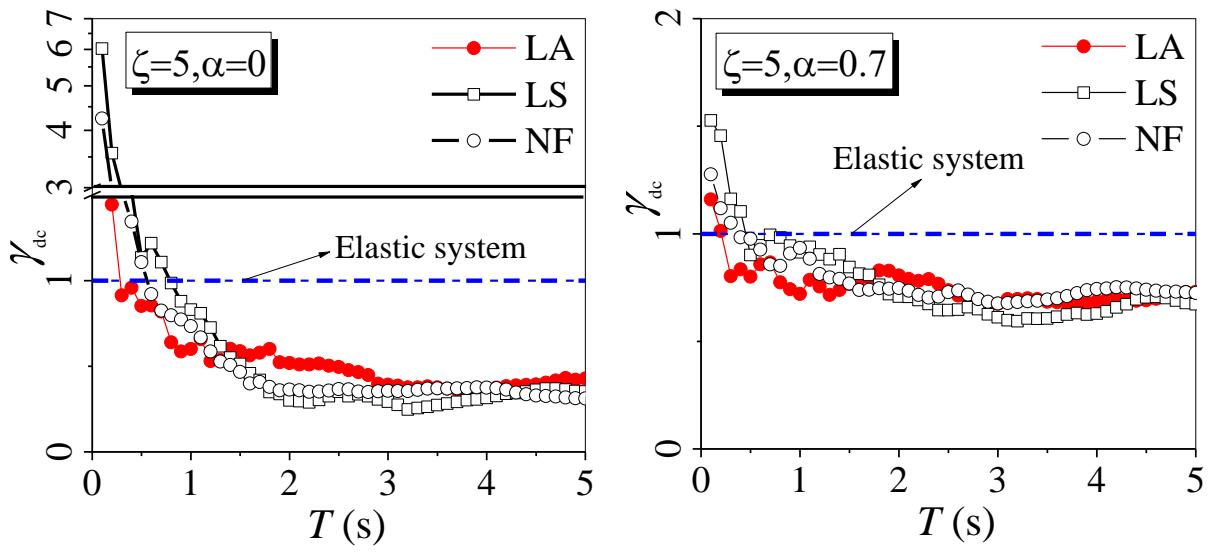

Fig. 8. Reduction of difference of the energy factor for systems with a DCC.
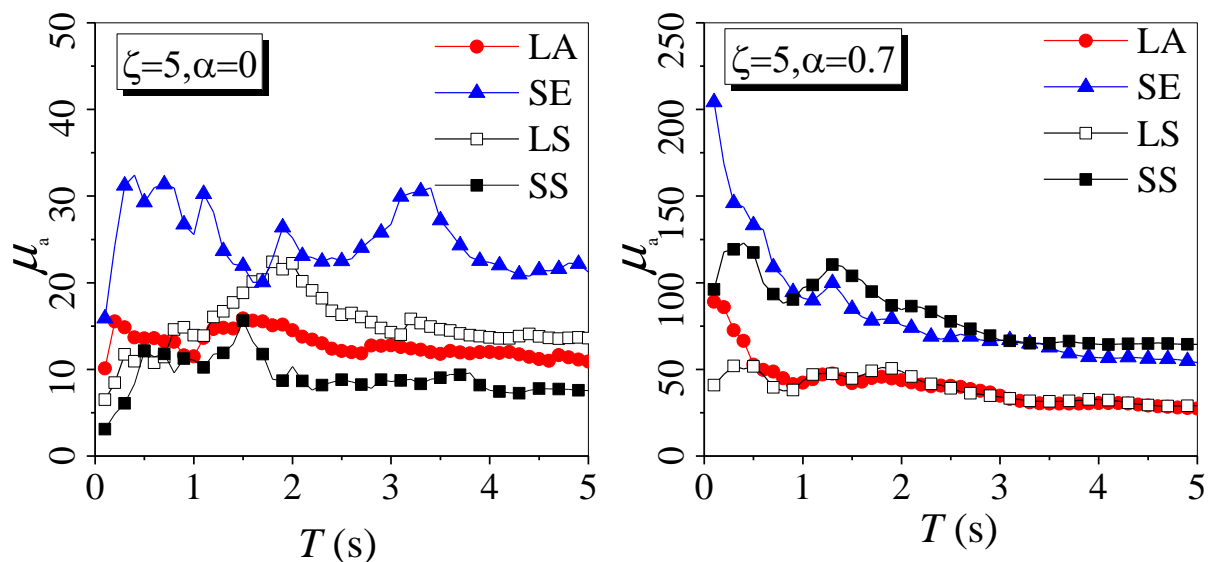

Fig. 9. Comparison of the cumulative ductility of EP systems and systems with a DCC. 

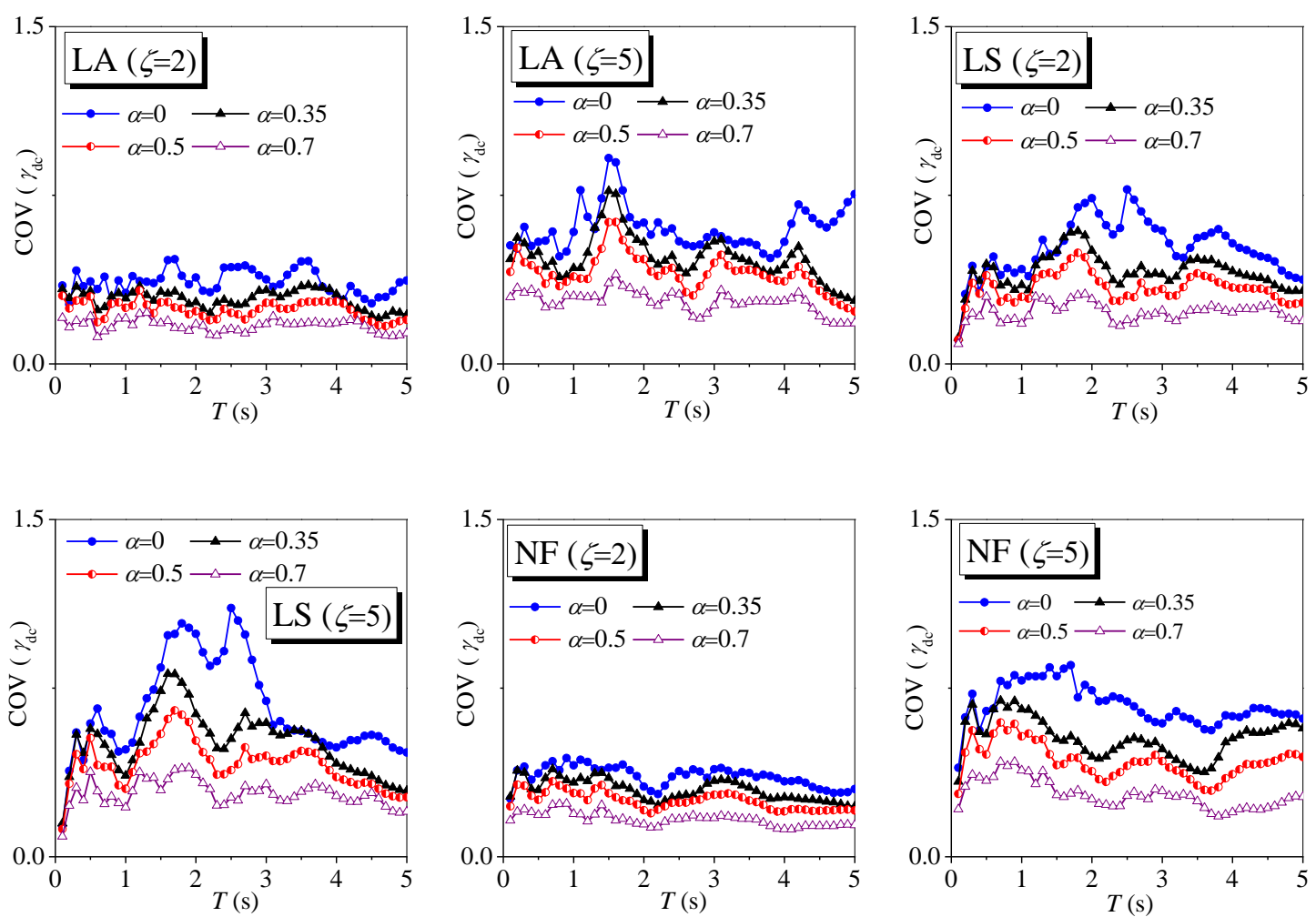

Fig. 10. Representative dispersion results of the energy factor.
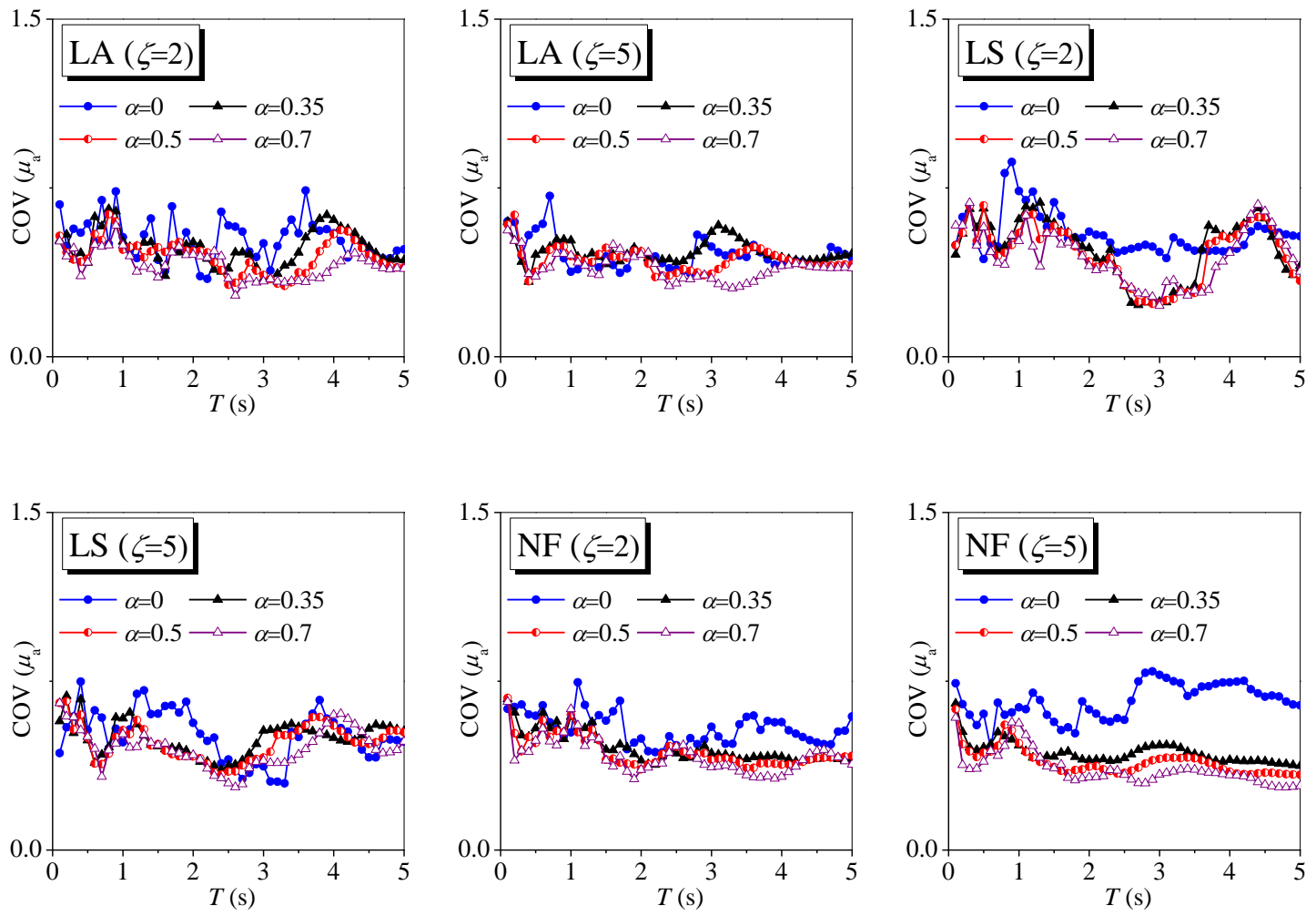

Fig. 11. Representative dispersion results of the cumulative ductility. 


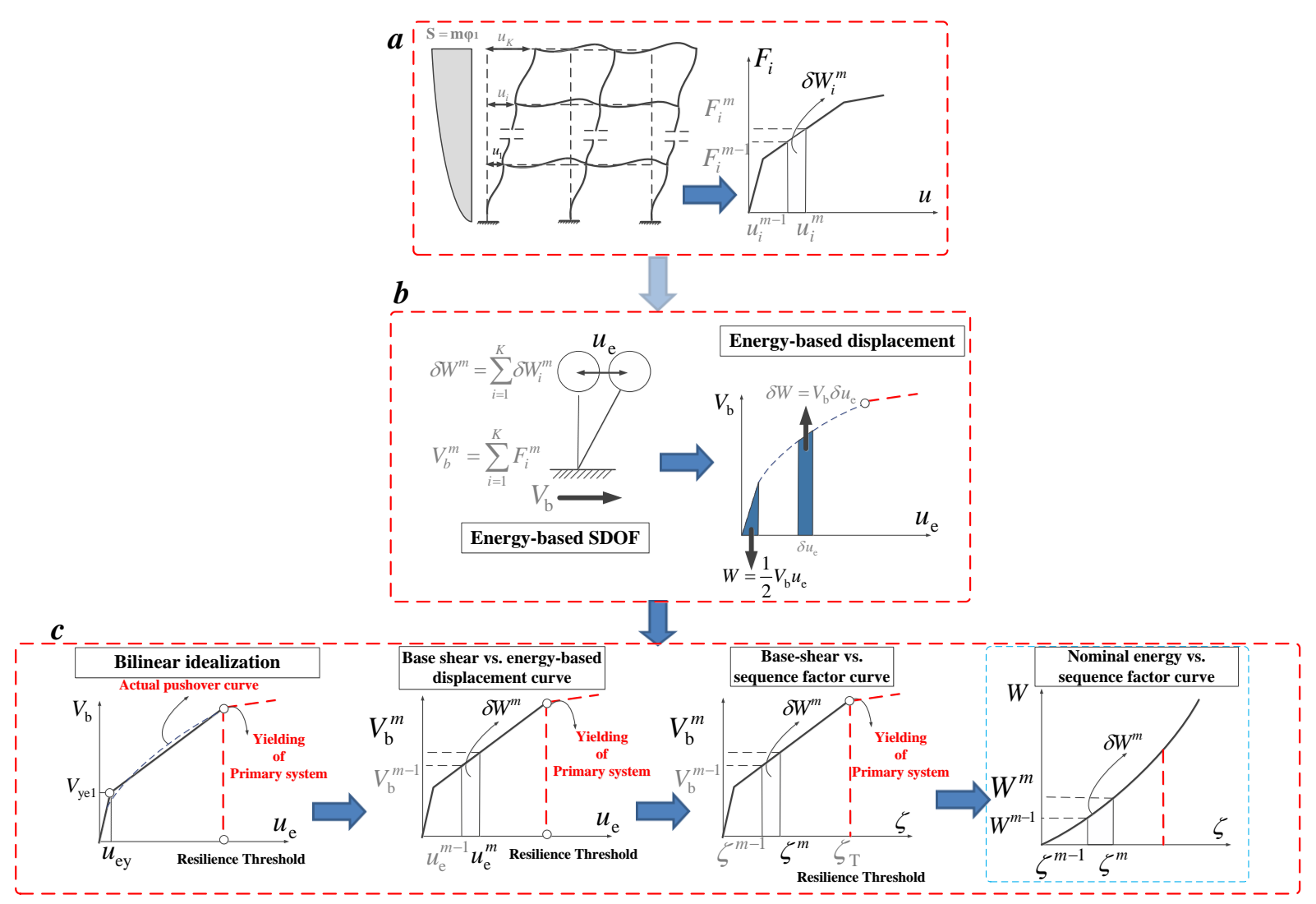

Fig. 12. Development of the nominal energy capacity diagram.
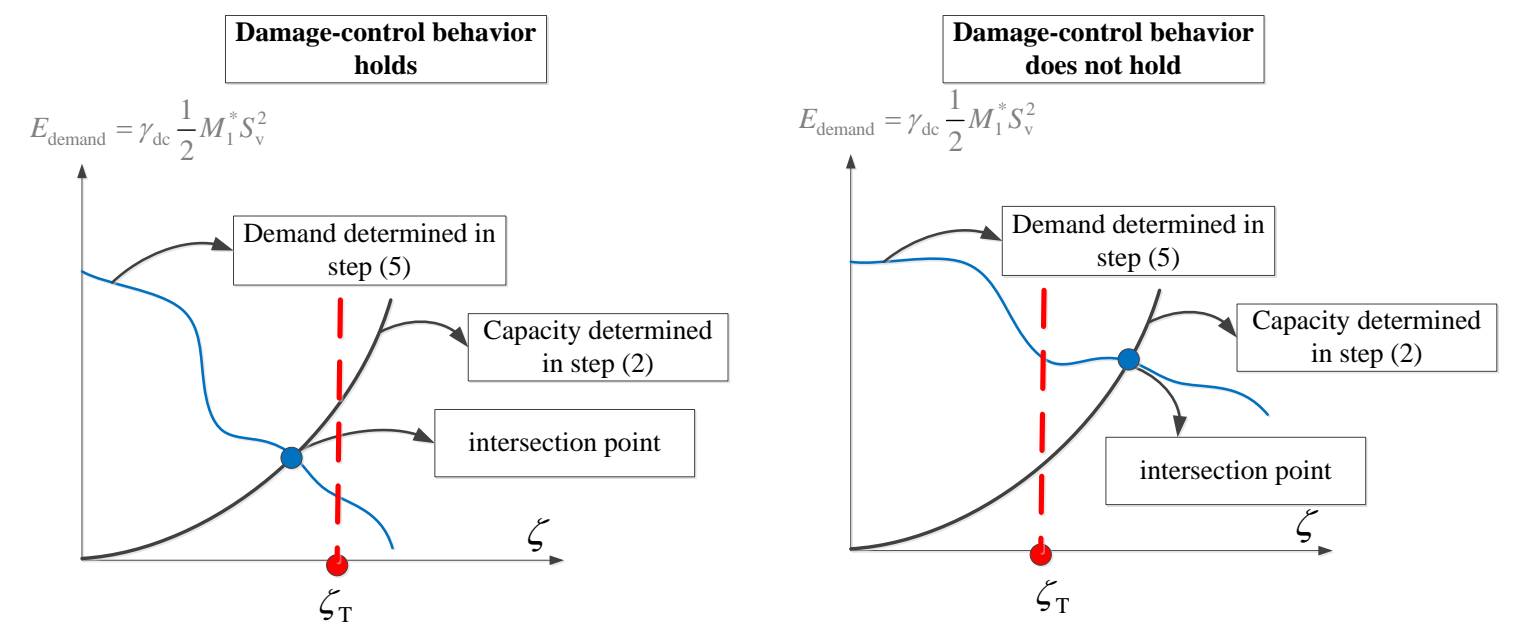

Fig. 13. Damage-control check by energy-balance concept (nominal energy demand and capacity). 


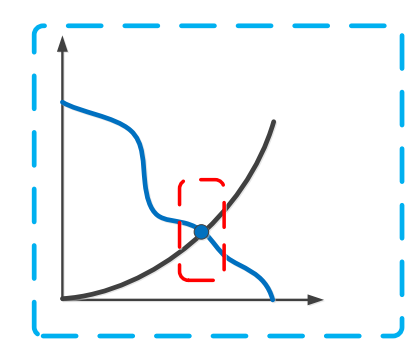

Apply the intersection point to determine the peak response

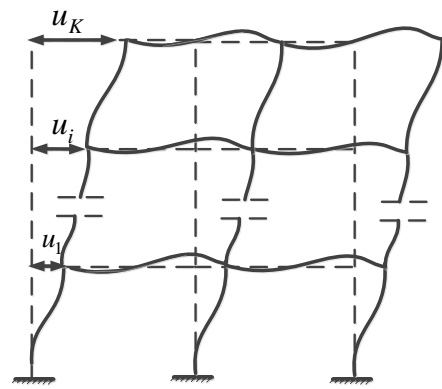

Extract the absorbed energy under pushover lateral loads at the peak deformation

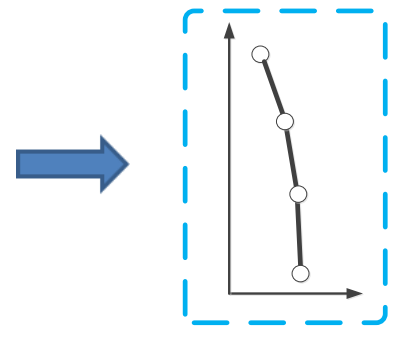

Determine the energy profile $[26,31]$

Fig.14. Energy profile determined by the energy balance.

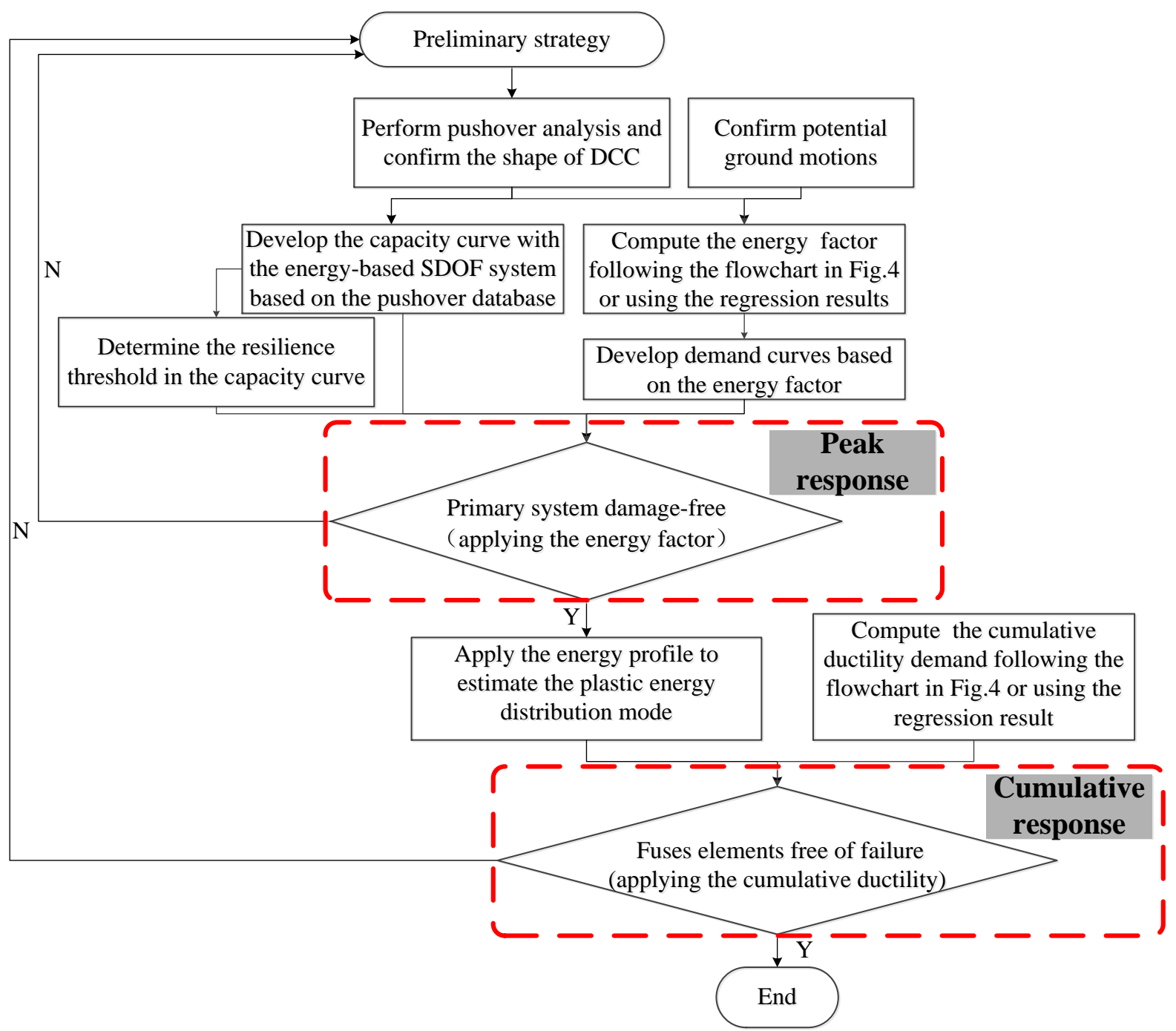

Fig. 15. Framework of DEB procedure. 
Frame A

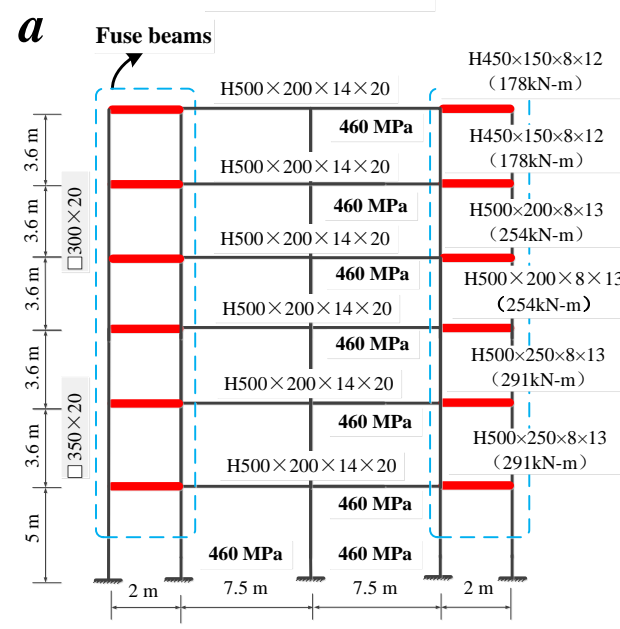

Frame B

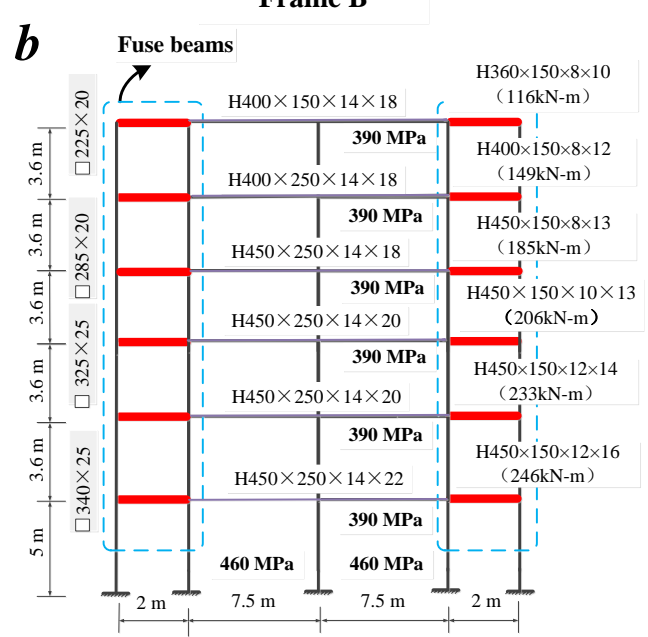

Fig. 16. Information on the prototype structures: (a) Frame A, (b) Frame B. 

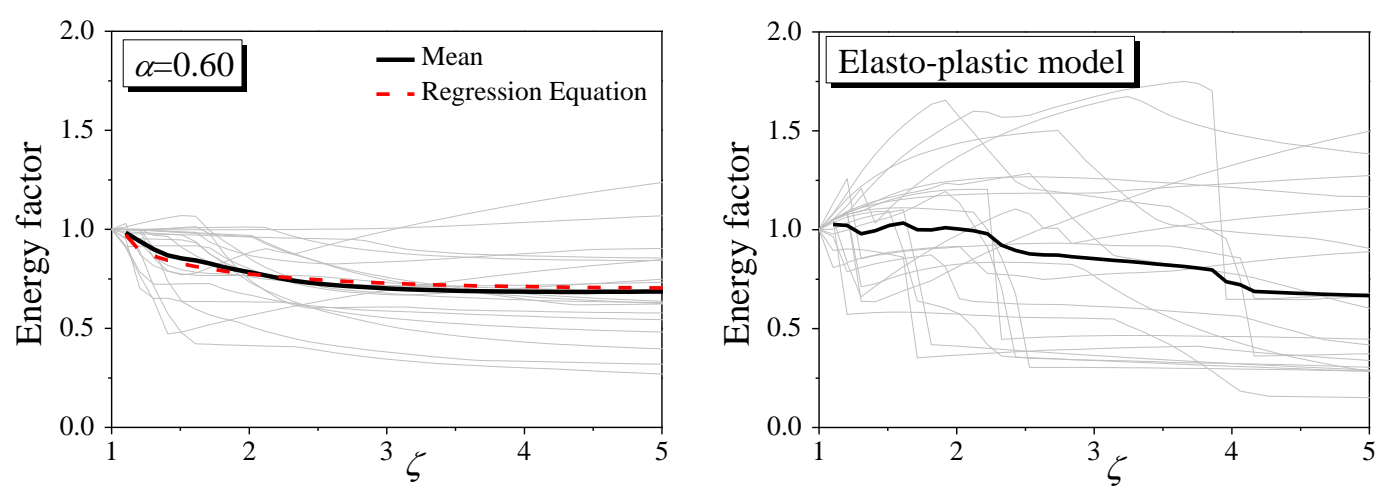

(a)
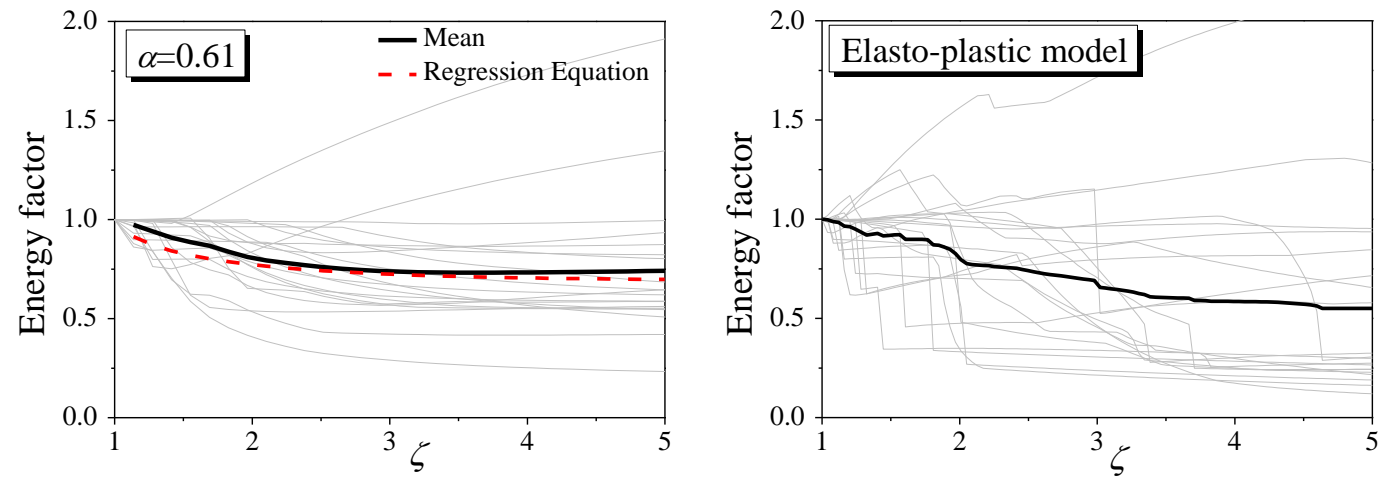

(b)

Fig. 17. Energy factor of the equivalent SDOF system of identical periods to frame A and frame B: (a) systems with DCC and EP systems for frame A, (b) systems with DCC and EP systems for frame B.
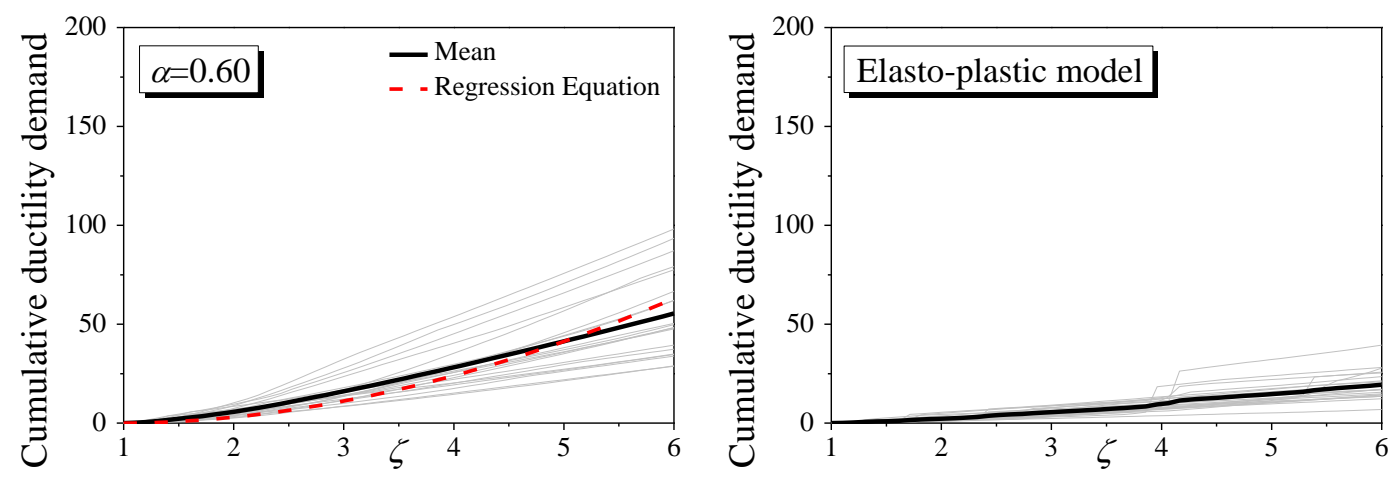

(a)
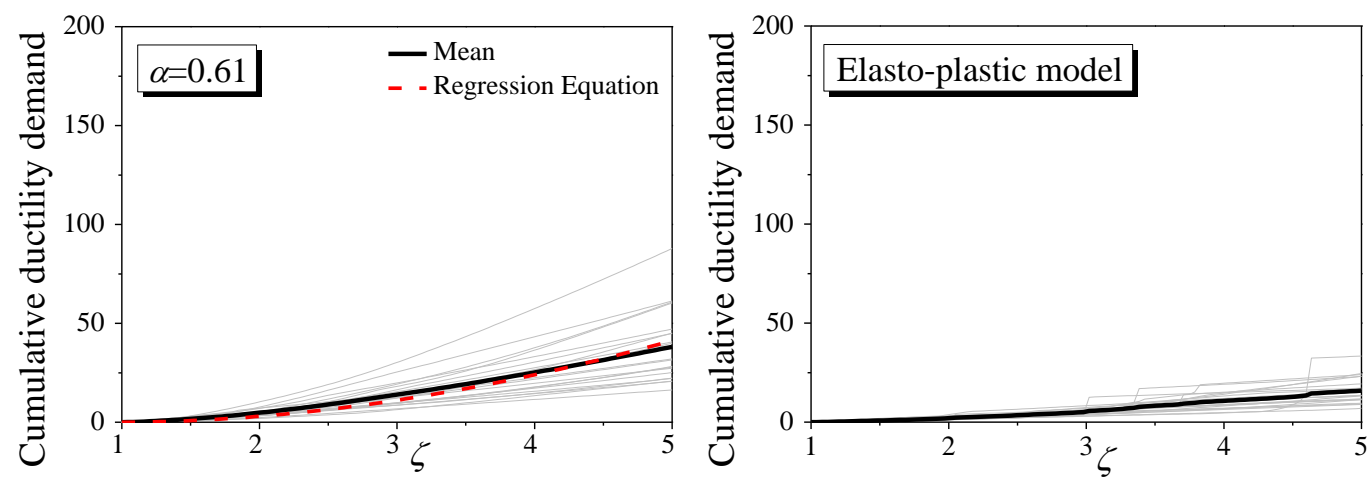

(b)

Fig. 18. Cumulative ductility of the equivalent SDOF system of identical periods to frame $A$ and frame B: (a) systems with DCC and EP systems for frame A, (b) systems with DCC and EP systems for 
frame B.
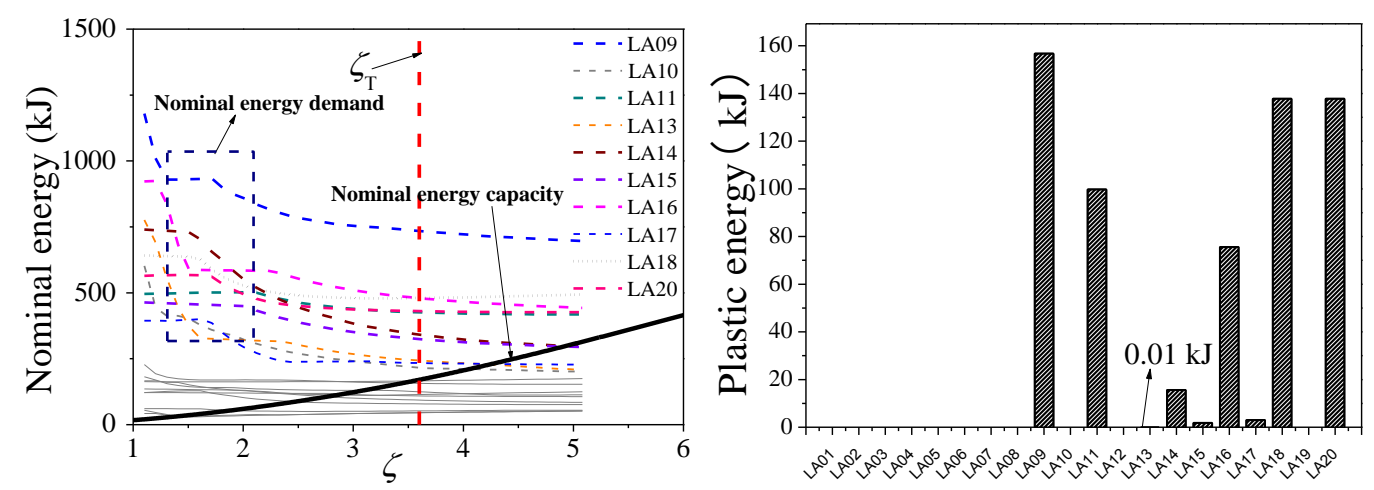

(a)
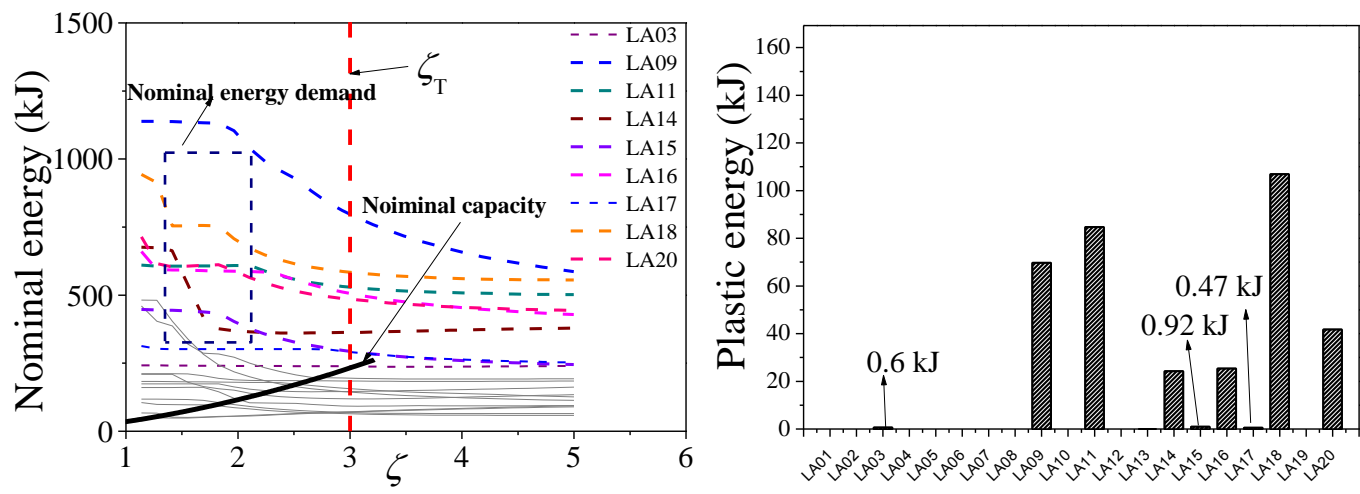

Fig. 19. Damage-control check based on demand and capacity curve and plastic energy dissipation of the primary system extracted from NL-RHA: (a) frame A, (b) frame B. 

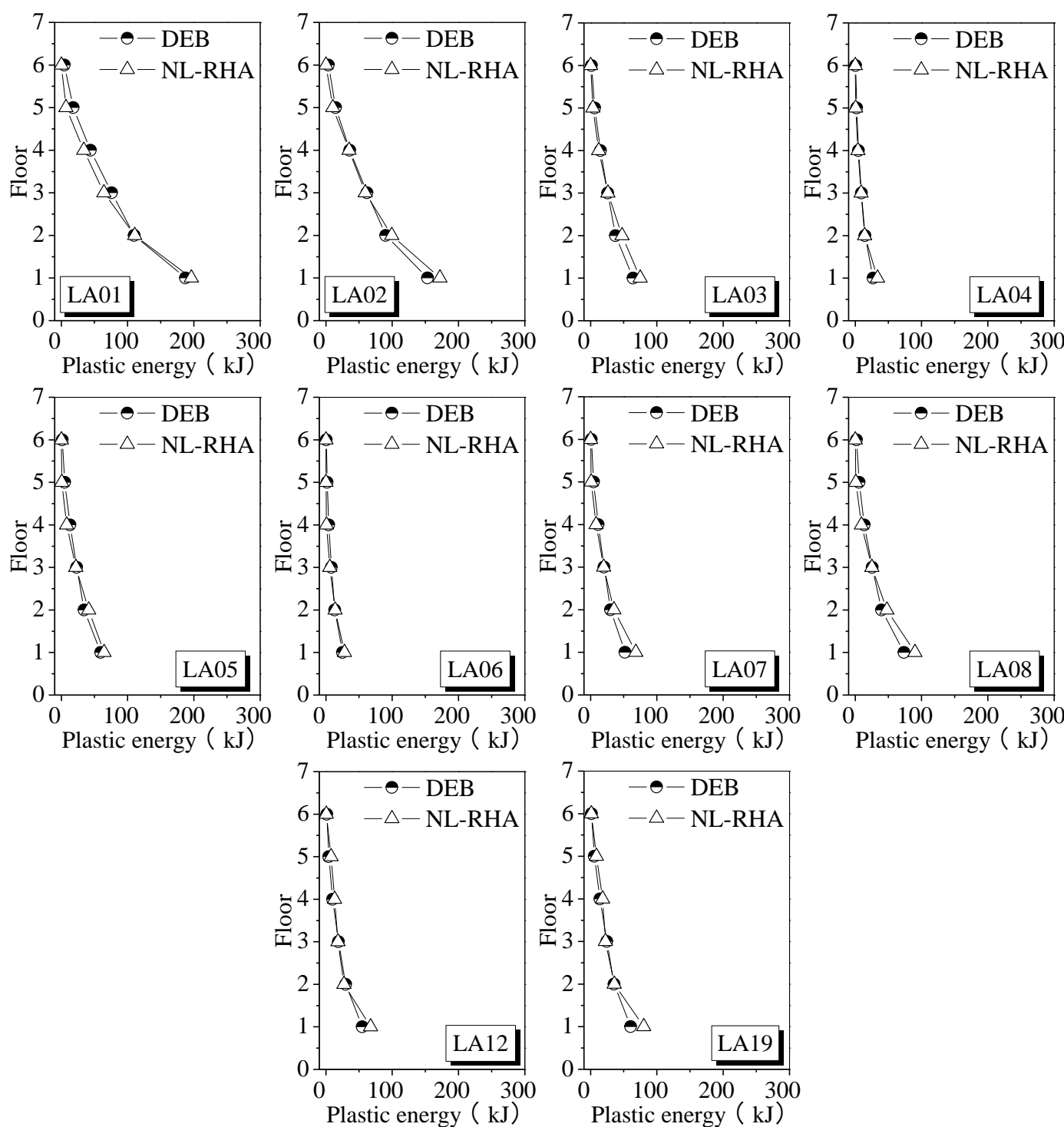

(a) 

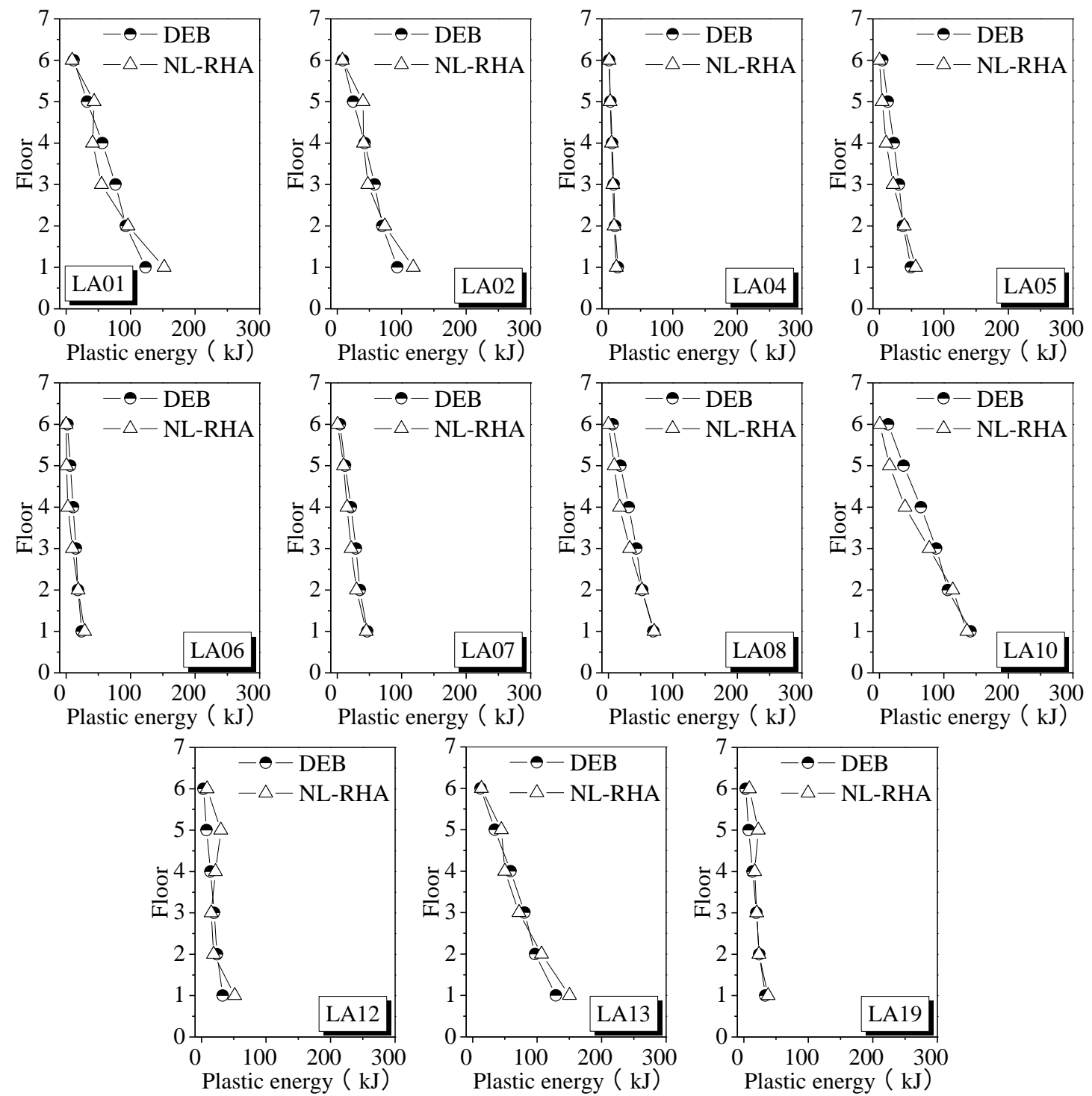

(b)

Fig. 20. Comparison of plastic energy distribution determined by different procedures: (a) frame A, (b) frame B. 

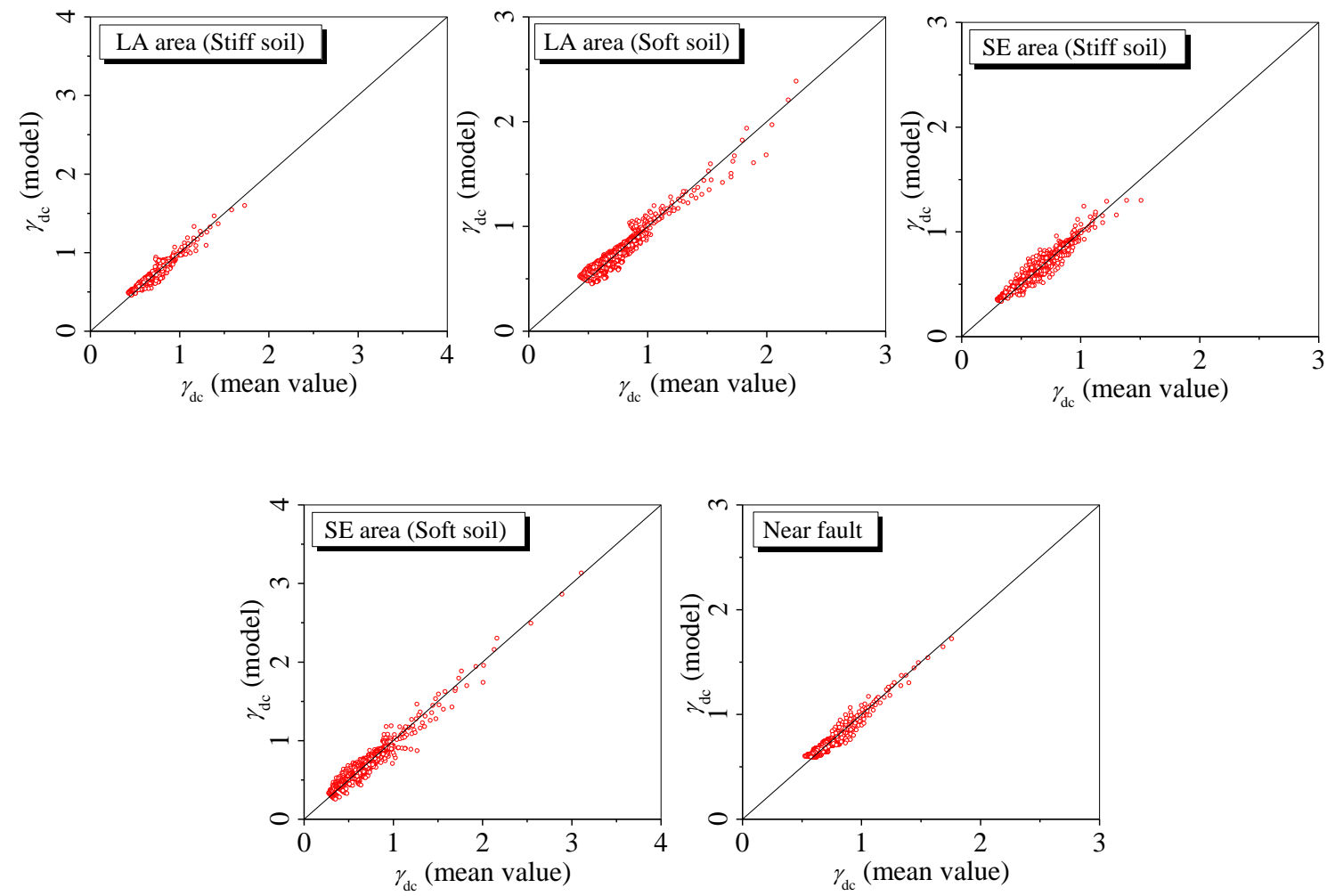

Fig. A1. Comparison of actual energy factor and regression results.
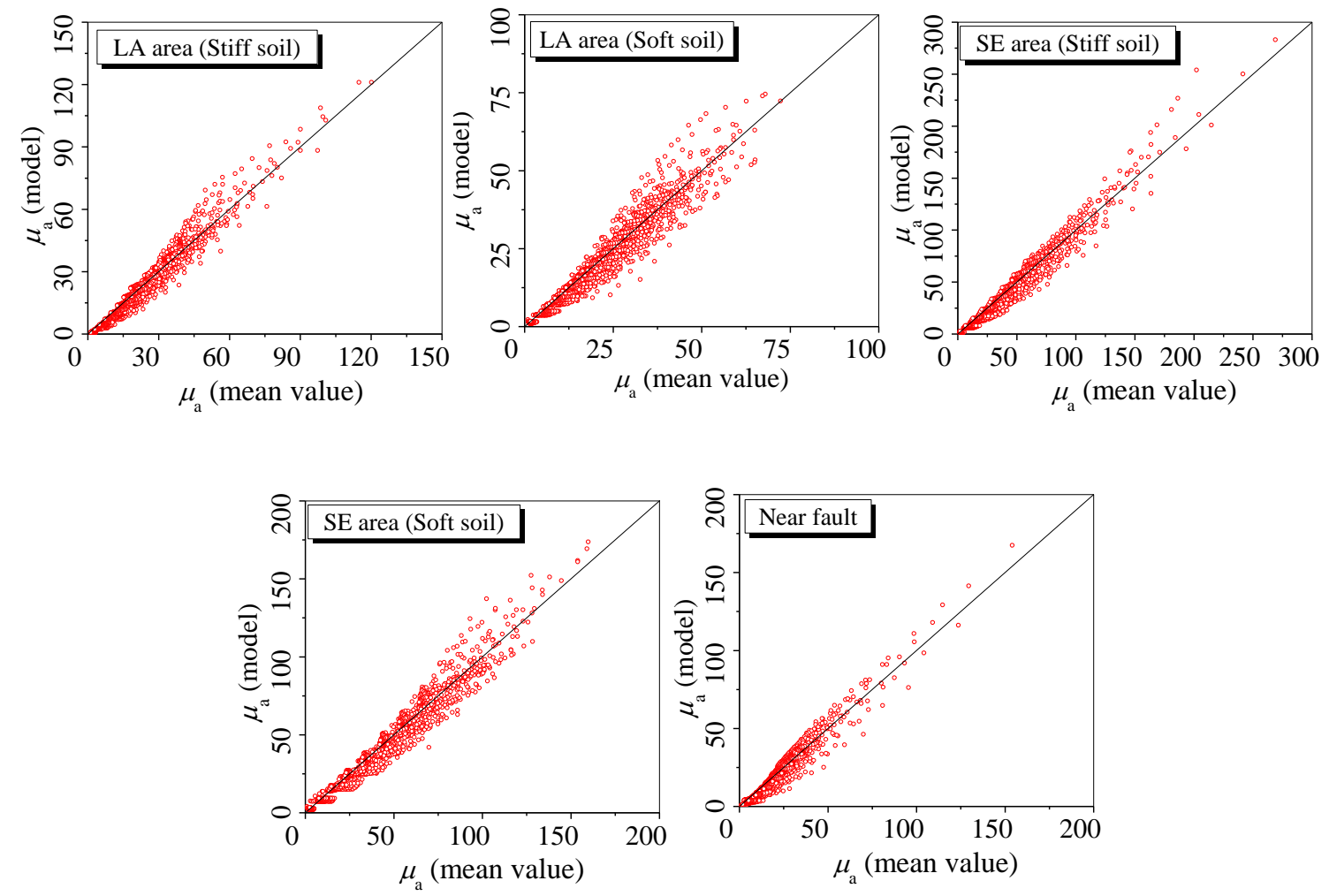

Fig. A2. Comparison of actual cumulative ductility and regression results. 
Table 1 Dynamic properties of the prototype structures.

\begin{tabular}{c|c|c}
\hline Structure & Property(unit) & $I^{\text {st }}$ Mode \\
\hline \multirow{3}{*}{ Frame A } & Period(s) & 1.35 \\
& Modal effective mass(t) & 471.3 \\
& Modal participation factor & 1.29 \\
\hline \multirow{3}{*}{ Frame B } & Period(s) & 1.52 \\
& Modal effective mass $(\mathrm{t})$ & 467.8 \\
& Modal participation factor & 1.34 \\
\hline
\end{tabular}


Table A1 Regression coefficients of the energy factor.

\begin{tabular}{|c|c|c|c|c|c|c|}
\hline \multirow{2}{*}{$\begin{array}{c}\text { Post-yield } \\
\text { stiffness ratio }\end{array}$} & \multirow{2}{*}{$\begin{array}{c}\text { Site } \\
\text { seismicity }\end{array}$} & \multirow[t]{2}{*}{ Site soil/Distance } & \multicolumn{4}{|c|}{ Regression coefficients } \\
\hline & & & $a$ & $b$ & $c$ & $d$ \\
\hline \multirow[t]{5}{*}{0.35} & Los Angeles & Stiff & 0.4678 & 0.5606 & -0.4669 & 1.1198 \\
\hline & & Soft & -0.0912 & 1.5127 & -1.1697 & 1.1178 \\
\hline & Seattle & Stiff & 0.4062 & 0.5291 & -0.5200 & 1.0660 \\
\hline & & Soft & -0.1920 & 1.6750 & -1.8587 & 1.0593 \\
\hline & & Near fault & 0.3105 & 0.9304 & -0.8183 & 1.1152 \\
\hline \multirow[t]{5}{*}{0.5} & Los Angeles & Stiff & 0.6173 & 0.3231 & -0.2176 & 1.1012 \\
\hline & & Soft & 0.3852 & 0.6354 & -0.1214 & 1.0935 \\
\hline & Seattle & Stiff & 0.5418 & 0.3228 & -0.2626 & 1.0643 \\
\hline & & Soft & 0.2536 & 0.8284 & -0.6782 & 1.0562 \\
\hline & & Near fault & 0.5843 & 0.4410 & -0.2366 & 1.0847 \\
\hline \multirow[t]{5}{*}{0.6} & Los Angeles & Stiff & 0.6909 & 0.2297 & -0.1418 & 1.0846 \\
\hline & & Soft & 0.5648 & 0.3608 & 0.1039 & 1.0769 \\
\hline & Seattle & Stiff & 0.6259 & 0.2251 & -0.1582 & 1.0571 \\
\hline & & Soft & 0.4471 & 0.5112 & -0.2957 & 1.0514 \\
\hline & & Near fault & 0.7002 & 0.2644 & -0.0747 & 1.0675 \\
\hline \multirow[t]{5}{*}{0.7} & Los Angeles & Stiff & 0.7591 & 0.1553 & -0.0842 & 1.0663 \\
\hline & & Soft & 0.6968 & 0.2006 & 0.1636 & 1.0596 \\
\hline & Seattle & Stiff & 0.7132 & 0.1337 & -0.0649 & 1.0473 \\
\hline & & Soft & 0.5894 & 0.3216 & -0.1231 & 1.0455 \\
\hline & & Near fault & 0.7892 & 0.1534 & -0.0069 & 1.0503 \\
\hline \multirow[t]{5}{*}{0.8} & Los Angeles & Stiff & 0.8260 & 0.0999 & -0.0549 & 1.0487 \\
\hline & & Soft & 0.8065 & 0.1021 & 0.1408 & 1.0403 \\
\hline & Seattle & Stiff & 0.7710 & 0.0875 & -0.0436 & 1.0825 \\
\hline & & Soft & 0.7172 & 0.1919 & -0.0462 & 1.0365 \\
\hline & & Near fault & 0.8627 & 0.0808 & 0.0148 & 1.0346 \\
\hline \multirow[t]{5}{*}{0.9} & Los Angeles & Stiff & 0.9051 & 0.0514 & -0.0353 & 1.0261 \\
\hline & & Soft & 0.9041 & 0.0408 & 0.0774 & 1.0203 \\
\hline & Seattle & Stiff & 0.8891 & 0.0318 & 0.0017 & 1.0216 \\
\hline & & Soft & 0.8477 & 0.0930 & -0.0185 & 1.0216 \\
\hline & & Near fault & 0.9309 & 0.0316 & 0.0126 & 1.0182 \\
\hline
\end{tabular}


Table A2 Regression coefficients of the cumulative ductility.

\begin{tabular}{|c|c|c|c|c|c|}
\hline \multirow{2}{*}{$\begin{array}{l}\text { Post-yielding } \\
\text { stiffness ratio }\end{array}$} & \multirow[t]{2}{*}{ Site seismicity } & \multirow[t]{2}{*}{ Site soil/Distance } & \multicolumn{3}{|c|}{ Regression coefficients } \\
\hline & & & $f$ & $g$ & $h$ \\
\hline \multirow[t]{5}{*}{0.35} & Los Angeles & Stiff & 2.2777 & 0.2769 & 0.01141 \\
\hline & & Soft & 3.733 & 0.6045 & 0.1188 \\
\hline & Seattle & Stiff & 5.5218 & 0.2732 & 0.0137 \\
\hline & & Soft & 7.4971 & 0.3021 & 0.0811 \\
\hline & & Near fault & 1.9814 & 0.3278 & 0.0106 \\
\hline \multirow[t]{5}{*}{0.5} & Los Angeles & Stiff & 2.6433 & 0.2824 & 0.0079 \\
\hline & & Soft & 3.6875 & 0.4724 & 0.0731 \\
\hline & Seattle & Stiff & 5.9889 & 0.3264 & 0.0119 \\
\hline & & Soft & 7.3657 & 0.3268 & 0.0477 \\
\hline & & Near fault & 2.3177 & 0.3367 & 0.00818 \\
\hline \multirow[t]{5}{*}{0.6} & Los Angeles & Stiff & 2.9975 & 0.2980 & 0.0084 \\
\hline & & Soft & 3.704 & 0.4015 & 0.0517 \\
\hline & Seattle & Stiff & 6.3738 & 0.3502 & 0.011 \\
\hline & & Soft & 7.3245 & 0.3230 & 0.0322 \\
\hline & & Near fault & 2.5724 & 0.3350 & 0.0067 \\
\hline \multirow[t]{5}{*}{0.7} & Los Angeles & Stiff & 3.4042 & 0.3032 & 0.0088 \\
\hline & & Soft & 3.9704 & 0.3876 & 0.0445 \\
\hline & Seattle & Stiff & 6.7967 & 0.3568 & 0.0098 \\
\hline & & Soft & 7.8013 & 0.3674 & 0.0304 \\
\hline & & Near fault & 2.8893 & 0.3382 & 0.00591 \\
\hline \multirow[t]{5}{*}{0.8} & Los Angeles & Stiff & 3.911 & 0.3135 & 0.0095 \\
\hline & & Soft & 4.3397 & 0.3797 & 0.0395 \\
\hline & Seattle & Stiff & 7.3424 & 0.3559 & 0.0087 \\
\hline & & Soft & 8.4663 & 0.4186 & 0.0313 \\
\hline & & Near fault & 3.2785 & 0.3409 & 0.0055 \\
\hline \multirow[t]{5}{*}{0.9} & Los Angeles & Stiff & 4.5713 & 0.3252 & 0.0102 \\
\hline & & Soft & 4.914 & 0.3791 & 0.0383 \\
\hline & Seattle & Stiff & 8.0825 & 0.3600 & 0.0086 \\
\hline & & Soft & 9.2844 & 0.4552 & 0.0321 \\
\hline & & Near fault & 3.7743 & 0.3404 & 0.0052 \\
\hline
\end{tabular}

